\title{
LÍNEA DE BASE COMPETENCIAS CIUDADANAS. RESULTADOS DE LA ENCUESTA CCO1
}

\author{
Paulo César Giraldo Betancur ${ }^{\mathrm{i}}$, Frandiney Henao Rios ${ }^{\mathrm{i}}$, Juan Pablo Jaramillo Salazar ${ }^{\mathrm{i}}$ \\ ,Yolanda Medina Bermudez ${ }^{i}$, Rocío Orozco Salazar ${ }^{i}, J u a n$ Carlos Osorio Santa ${ }^{i}$ \\ ${ }^{i}$ Profesionales de CONFAMILIARES E-mail saturno153@yahoo.com.ar
}

Manizales, 2008-10-01 (Rev. 2008-04-14)

\section{RESUMEN}

El presente artículo entrega los resultados finales obtenidos a partir del levantamiento de una línea de base en el tema de ciudadanía durante el año 2006, resultado de la incorporación de la investigación social al proyecto de Competencias Ciudadanas: "Ciudadanos con todo y con todos", desarrollado desde el año 2004 y hasta al fecha por la Caja de compensación familiar de Caldas, CONFAMILIARES.

Los resultados aquí expuestos y las generalizaciones posibles en el análisis, aplican al contexto educativo de la básica secundaria y la media del Departamento de Caldas, ya que los grupos a encuestar, resultado del muestreo, se distribuyeron en instituciones públicas de la mayoría de sus Municipios. Estos resultados son aplicables además al contexto de trabajadores vinculados a la Caja de Compensación, sin excluir que las generalizaciones contenidas, pueden guardar relaciones cercanas con mediciones que en este mismo tema y para el mismo grupo (colaboradores de empresas y ciudadanos), han elaborado el PNUD y otras entidades de investigación del Departamento. Para realizar el estudio diagnóstico mencionado se ejecutaron aproximadamente 230 encuestas divididas, en los dos grupos. Como modelo metodológico se utilizó la experiencia vivida en la cuidad de Bogotá, concordante con el periodo de gobierno del Alcalde Antanas Mocus, además de las mediciones ejecutadas en las ciudades de Cali y Medellín.

\section{PALABRAS CLAVE}

Ciudadanía, espacio público, participación social, participación política.

\section{CITIZENSHIP COMPETENCIES BASELINE. RESULTS OF THE CCO1 SURVEY}

\begin{abstract}
This article delivers the final results obtained from the baseline on the issue of citizenship in 2006, resulting from the incorporation of social research to project entitled Citizenship Competences: "Citizens with everything and everybody", developed since 2004 and up to the present by the Family Compensation Fund of Caldas, CONFAMILIARES. The results presented here and the generalizations possible in the
\end{abstract}


analysis, applied to the educative context of basic secondary and middle education of the Department of Caldas, since the groups surveyed, resulting from sampling, were distributed in public institutions of the majority of its Municipalities. These findings are also applicable to the context of workers linked to the Compensation Fund, without excluding that contained generalizations can be closely related to measurements on the same subject and for the same group (business partners and citizens), developed by the UNDP and other research entities of the Caldas. In order to carry out abovementioned diagnosis study approximately 230 surveys were executed divided into two groups. The experience in the city of Bogota was used as a methodological model, consistent with the governing period of the Mayor Antanas Mocus, as well as measurements performed in the cities of Cali and Medellin.

\section{KEY WORDS}

Citizenship, public space, social participation, political participation.

\section{INTRODUCCIÓN}

El presente artículo muestra los principales resultados cualitativos y cuantitativos del estudio diagnostico de Línea de Base que la Caja de Compensación Familiar de Caldas, CONFAMILIARES, desde su Proyecto de Competencias Ciudadanas "Ciudadanos con todo y con todos", elaboró en el año 2005. El propósito de éste fue el reconocimiento de las principales percepciones y tendencias acerca de las variables constituyentes de la ciudadanía y la convivencia social en el departamento de Caldas.

El estudio midió, a través de encuesta por muestreo, dimensiones de la ciudadanía tales como la solidaridad, la tolerancia, la identidad, el sentido de pertenencia frente al patrimonio público y los lugares de residencia, la confianza interpersonal y en las instituciones; además de analizar sus respectivos indicadores en estudiantes de Décimo y Undécimo de colegios públicos y trabajadores de empresas privadas del departamento de Caldas.

El aporte de esta publicación es el de democratizar la información para su uso por parte de Instituciones Gubernamentales, Organizaciones de la Sociedad Civil y Organizaciones no Gubernamentales con el fin de que cuenten con estudios rigurosos desde la investigación científica que les facilite la toma de decisiones informadas para la intervención en el desarrollo humano desde su esfera ética y política entendida como la generación de habilidades para el ejercicio de la convivencia, la pluralidad y la participación, tanto en los ciudadanos como en las iniciativas y movimientos a los que pertenecen.

El texto es además una evidencia y un resultado intermedio desde una Organización Sin Ánimo de Lucro para conducir desde la cultura aquellos necesarios pasos que la sociedad colombiana está dando hacia la consolidación de estrategias más efectivas que las actuales para la promoción de una visión compartida de la ciudadanía y la 
convivencia, en contextos que cada día más exploran alternativas a la negociación de sus controversias y a la vida en común.

El estudio del que deriva el documento presente ha servido en el contexto del proyecto Corporativo de Competencias Ciudadanas a la reprogramación y la mejora de la calidad de las intervenciones en las comunidades educativas, de trabajadores y de la ciudad en general, expresadas en estrategias de formación y comunicación que oferta la Caja de Compensación como aporte subsidiado al mejoramiento de los niveles de convivencia y democracia de la sociedad caldense y el país.

El reto para los gobiernos locales y centrales, las entidades de Cooperación, la Organizaciones de la Sociedad Civil y la Academia es el de vincular a sus planes de gestión de la política pública social iniciativas de formación para la democracia y la cultura ciudadana desde un enfoque de corresponsabilidad y como fundamento de su proyección social y cuidado con los ciudadanos.

\section{JUSTIFICACIÓN}

La encuesta $\mathrm{CC} 01$ del proyecto de competencias ciudadanas pretende ser un resultado intermedio. Es solamente un paso hacia la consolidación de las estrategias más efectivas para promover una visión de la ciudadanía que nos pretenda tener una sociedad más amable. También son intermedios sus resultados, en la medida en que son un puente hacia varios aspectos que enfrenta el proyecto:

1. Un puente hacia la consolidación de indicadores que trasciendan las coberturas, en una labor tan ardua como cambiar la cultura.

2. Un puente hacia estudios que profundicen todos los vacíos que dejan estos resultados y que sólo pueden ser abordados por estrategias más etnográficas. Estos estudios deben ser también iniciativa dentro del proyecto y no dejar esta responsabilidad a otros.

3. Un puente hacia mejores comprensiones de las tramas del gran tapiz que llamamos ciudadanía.

A pesar de no querer otra cosa que cantar victoria con estos resultados, pedimos mesura y empeño frente a ellos. Mesura, porque como pronto comprenderá el lector inteligente hay más preguntas que respuestas en estas páginas. Empeño porque es una piedra en bruto aún. Es necesario desbastarla y tallarla, pero también resanarla y restaurarla cuando el tiempo o las manos de los observadores (como quienes escriben) hayan quebrado la utilidad de los resultados.

\section{PROBLEMAS METODOLÓGICOS}

Es importante no omitir algunos problemas metodológicos que ayudan a valorar los alcances de los resultados presentados. En primer lugar, en particular con los grupos de empresas y de bajos niveles educativos se presentaron dificultades en la respuesta de 
preguntas que pretendían indagar sobre percepciones a través de puntajes del 1 al 5 , en este caso.

Por otro lado, con algunas poblaciones (como Mineros Nacionales) donde el analfabetismo es muy alto, no fue posible aplicar la encuesta. Obviamente esto da un sesgo a la misma y es que aun existiendo población analfabeta, la encuesta no estaba diseñada para este tipo de población. También nos da un indicativo sobre un menor alcance de los resultados en términos de empresas debido a la misma heterogeneidad de este grupo.

En el caso de los estudiantes, la encuesta es bastante confiable y se pueden hacer aseveraciones de alcance departamental sobre los estudiantes de colegios públicos, hombres y mujeres con una edad promedio de 16 años.

\section{RESULTADOS DE LA ENCUESTA}

\section{ESTRATIFICACIÓN}

Los colaboradores de las empresas afiliadas (CEA) pertenecen en un 50\% al estrato 3, seguido de un $21.3 \%$ que viven en estrato 2 . El $18.52 \%$ vive en estratos 1 ó 4 . Por otro lado, los estudiantes (E) pertenecen en un $52.8 \%$ al estrato 2 y un $32.8 \%$ al estrato 3 . Entre esta población no hay casos de estudiantes que provengan de estratos 5 ó 6 . Un factor importante para analizar los estratos en este estudio en particular, es la gran representación de personas habitantes de municipios diferentes a Manizales, donde la composición por estratos se concentra en estratos 2 y 3 y no existen barrios de estrato 5 ó 6 .

\section{Empresas}

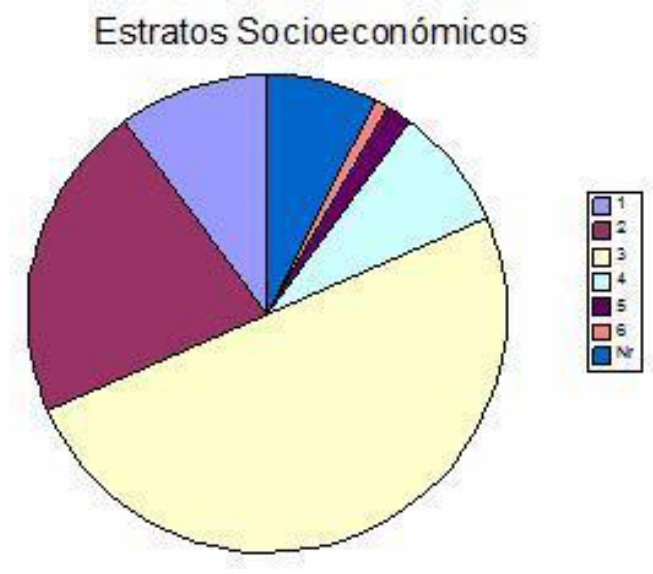

\begin{tabular}{|l|l|}
\hline Estrato Socioeconómico & $\%$ \\
\hline 1 & 10.19 \\
\hline 2 & 21.3 \\
\hline 3 & 50 \\
\hline
\end{tabular}




\begin{tabular}{|l|l|}
\hline 4 & 8.33 \\
\hline 5 & 1.85 \\
\hline 6 & 0.93 \\
\hline $\mathrm{Nr}$ & 7.41 \\
\hline
\end{tabular}

\section{Estudiantes}

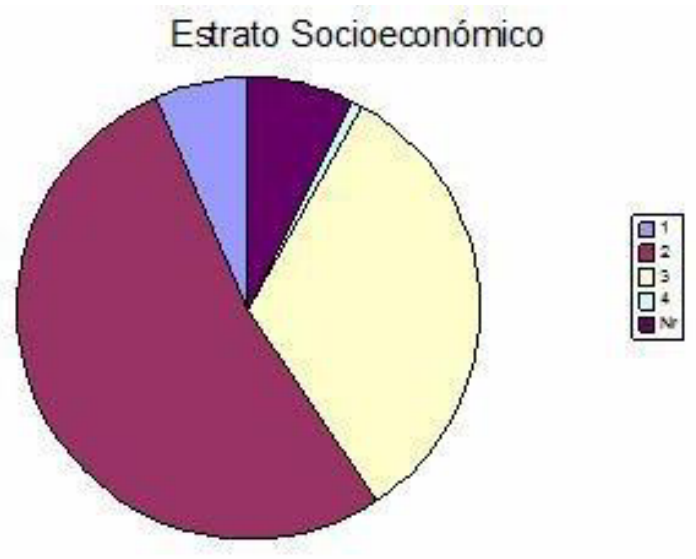

\begin{tabular}{|l|l|}
\hline Estrato socioeconómico & $\mathbf{\%}$ \\
\hline 1 & 6.4 \\
\hline 2 & 52.8 \\
\hline 3 & 32.8 \\
\hline 4 & 0.8 \\
\hline $\mathrm{Nr}$ & 7.2 \\
\hline Total & 100 \\
\hline
\end{tabular}

Es importante tener en cuenta que los estudiantes que se incluyeron en el estudio pertenecen a instituciones educativas que hacen parte del programa Jornada Escolar Complementaria que tiene como uno de sus requisitos ser una institución pública. La estratificación entre estudiantes tiende a ser, por lo tanto, un reflejo de la composición de las instituciones educativas públicas.

\section{SEXO}

Tanto en E como en CEA, la población se encuentra des-balanceada en términos de sexo. Los estudiantes tienen una ligera inclinación hacia el sexo masculino con un $52 \%$ y con un $48 \%$ el femenino.

\section{Estudiantes}




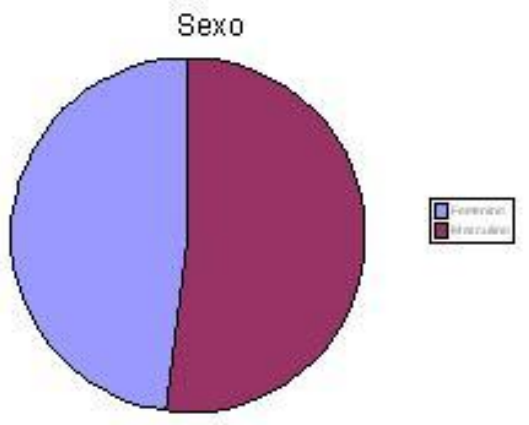

\begin{tabular}{|l|l|}
\hline Estudiantes & $\boldsymbol{\%}$ \\
\hline Femenino & 48 \\
\hline Masculino & 52 \\
\hline Total & 100 \\
\hline
\end{tabular}

Las empresas tienen un desbalance mucho mayor hacia la población masculina con un $57.41 \%$ y un $42.59 \%$ femenina.

\begin{tabular}{|l|l|}
\hline Empresas & \% \\
\hline Femenino & 42.59 \\
\hline Masculino & 57.41 \\
\hline Total & 100 \\
\hline
\end{tabular}

Un balance mayor entre la población de estudiantes es explicado por las políticas de ingreso a los colegios. Por otro lado, el desbalance existente entre empresas es causado por el carácter de las empresas incluidas en el proceso. Lo anterior implica que para este punto en particular la muestra no refleja la composición de los afiliados a las empresas de la caja.

\section{Estudiantes}

\begin{tabular}{|l|l|}
\hline Estado civil & $\mathbf{\%}$ \\
\hline Soltero & $96>$ \\
\hline Casado o en unión libre & 3.2 \\
\hline $\mathrm{Nr}$ & 0.8 \\
\hline
\end{tabular}

Estudiantes

\begin{tabular}{|l|l|}
\hline Cabeza de familia & $\%$ \\
\hline $\mathrm{Si}$ & 0.8 \\
\hline
\end{tabular}




\begin{tabular}{|l|l|}
\hline No & 97.6 \\
\hline $\mathrm{Nr}$ & 1.6 \\
\hline Total & 100 \\
\hline
\end{tabular}

\section{NIVEL EDUCATIVO}

Las dos poblaciones estudiadas son muy diferentes entre sí. Mientras que los estudiantes todos tienen secundaria incompleta por obvios motivos, los colaboradores de empresas afiliadas tienen en un $51.85 \%$ un nivel educativo de secundaria. Además, un porcentaje importante detenta títulos técnicos o universitarios con un $9.26 \%$ y un $20.37 \%$ respectivamente. Sólo un $13.89 \%$ tiene un nivel inferior a secundaria. En este sentido las estrategias de formación que actualmente se usan, enfocadas en un nivel medio y medio alto de nivel educativo, se encuentran ajustadas a las características de la población.

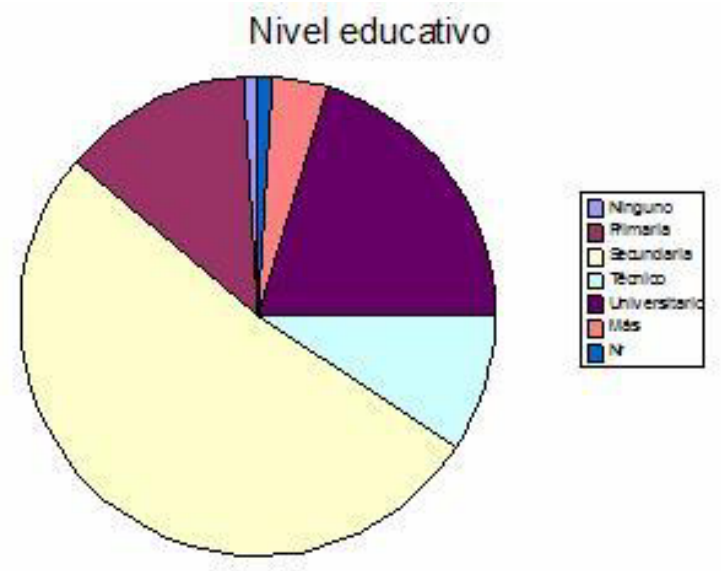

\begin{tabular}{|l|l|}
\hline Nivel educativo & $\%$ \\
\hline Ninguno & 0.93 \\
\hline Primaria & 12.96 \\
\hline Secundaria & 51.85 \\
\hline Técnico & 9.26 \\
\hline Universitario & 20.37 \\
\hline Más & 3.7 \\
\hline $\mathrm{Nr}$ & 0.93 \\
\hline
\end{tabular}

\section{PERCEPCIONES DEL BARRIO Y LA CIUDAD}

En términos generales, las percepciones frente al barrio son positivas entre ambos grupos. Derivando un indicador de las posiciones "positiva" (4) y "muy positiva" (5), tenemos para el caso de las empresas el $69.44 \%$ y de los estudiantes el $70.2 \% \underline{1}$.

\section{Percepción barrio- Empresas}




\begin{tabular}{|l|l|l|l|l|l|l|l|}
\hline$(-)$ & $\mathbf{1}$ & $\mathbf{2}$ & $\mathbf{3}$ & $\mathbf{4}$ & $\mathbf{5}$ & $\mathbf{( + )}$ & $\mathbf{N r}$ \\
\hline Inseguro & 0.93 & 2.78 & 19.44 & 39.81 & 28.7 & Seguro & 8.33 \\
\hline Desordenado & 0 & 5.56 & 16.67 & 41.67 & 21.3 & Ordenado & 12.81 \\
\hline Desagradable & 0.93 & 2.78 & 4.63 & 33.33 & 47.22 & Agradable & 11.11 \\
\hline Sucio & 1.85 & 2.78 & 12.96 & 32.41 & 33.33 & Limpio & 16.67 \\
\hline
\end{tabular}

\section{Percepción barrio- Estudiantes}

\begin{tabular}{|l|l|l|l|l|l|l|l|}
\hline$(-)$ & 1 & 2 & 3 & 4 & 5 & $(+)$ & $\mathrm{Nr}$ \\
\hline Inseguro & 1.6 & 8 & 20 & 32 & 37.6 & Seguro & 0.8 \\
\hline Desordenado & 0 & 6.4 & 28 & 36.8 & 26.4 & Ordenado & 2.4 \\
\hline Desagradable & 4.8 & 4.8 & 12 & 28.8 & 48.8 & Agradable & 0.8 \\
\hline Sucio & 2.4 & 2.4 & 22.4 & 35.2 & 35.2 & Limpio & 2.4 \\
\hline
\end{tabular}

A diferencia de las percepciones frente al barrio, las percepciones frente al municipio son mucho menos positivas. Utilizando el mismo modelo de indicador para las percepciones sobre el barrio, tenemos el $53.01 \%$ para colaboradores de empresas y el $52.2 \%$ para los estudiantes. En este caso, encontramos una gran franja de opinión concentrada en una posición intermedia predominantemente en 3 y en 4 . El problema que tiende a ser más señalado es seguridad, tema frente al cual el proyecto tiene limitadas posibilidades de acción. Es importante enfatizar en el trabajo frente a una visión intermedia del municipio.

\section{Percepción ciudad- Empresas}

\begin{tabular}{|l|l|l|l|l|l|l|l|}
\hline$(-)$ & 1 & 2 & 3 & 4 & 5 & $(+)$ & $\mathrm{Nr}$ \\
\hline Inseguro & 7.41 & 10.19 & 37.04 & 28.7 & 6.48 & Seguro & 10.19 \\
\hline Desordenado & 1.85 & 2.78 & 28.7 & 39.81 & 9.26 & Ordenado & 17.59 \\
\hline Desagradable & 0.93 & 2.78 & 12.04 & 37.04 & 36.11 & Agradable & 11.11 \\
\hline Sucio & 1.85 & 2.78 & 21.3 & 39.81 & 14.81 & Limpio & 19.44 \\
\hline Elitista & 2.78 & 6.48 & 23.15 & 37.96 & 14.04 & Popular & 17.59 \\
\hline
\end{tabular}

\section{Percepción ciudad- Estudiantes}

\begin{tabular}{|l|l|l|l|l|l|l|l|}
\hline$(-)$ & 1 & 2 & 3 & 4 & 5 & $(+)$ & $\mathrm{Nr}$ \\
\hline Inseguro & 8 & 13.6 & 46.4 & 22.4 & 8 & Seguro & 1.6 \\
\hline Desordenado & 1.6 & 8.8 & 39.2 & 33.6 & 15.2 & Ordenado & 1.6 \\
\hline Desagradable & 1.6 & 4.8 & 19.2 & 37.6 & 35.2 & Agradable & 1.6 \\
\hline
\end{tabular}




\begin{tabular}{|l|l|l|l|l|l|l|l|}
\hline Sucio & 0.8 & 8.8 & 32 & 38.4 & 18.4 & Limpio & 1.6 \\
\hline Elitista & 8.8 & 10.4 & 23.2 & 25.6 & 29.6 & Popular & 3.2 \\
\hline
\end{tabular}

\section{PROBLEMAS SENTIDOS}

Frente a este aspecto, en el cual el proyecto tiene limitadas posibilidades de acción, el problema más sentido entre las personas a las que se les aplicó la encuesta es el del empleo señalado por el $81.48 \%$ de los colaboradores de empresas afiliadas y el $95.2 \%$ de los estudiantes. Los siguientes problemas señalados son la seguridad y la falta de civismo con el $41.67 \%$ y $38.89 \%$ para los colaboradores de empresas y el $46.4 \%$ y el $42.8 \%$ para estudiantes respectivamente. Dos elementos particularmente señalados por los estudiantes es la contaminación (48\%) y la corrupción (43.2\%).

\section{Empresas}

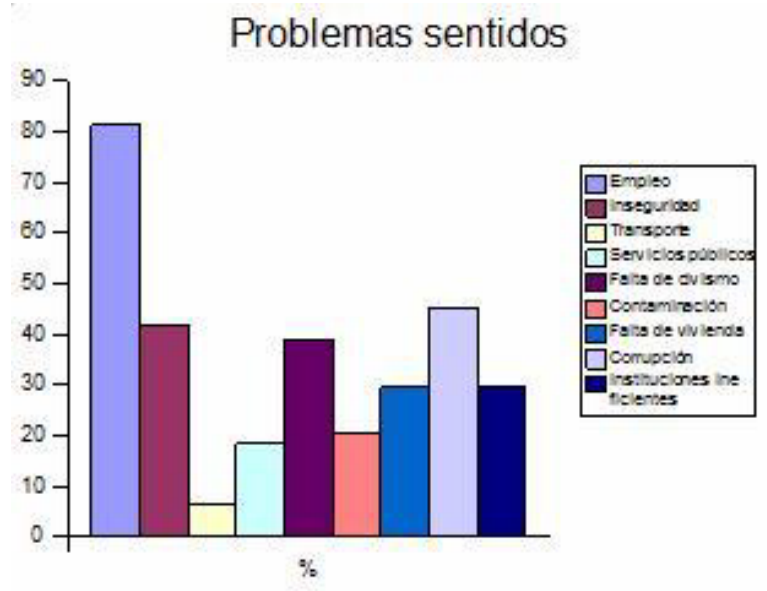

\begin{tabular}{|l|l|}
\hline Problemas sentidos & $\%$ \\
\hline Empleo & 81.48 \\
\hline Inseguridad & 41.67 \\
\hline Transporte & 6.48 \\
\hline Servicios públicos & 18.52 \\
\hline Falta de civismo & 38.89 \\
\hline Contaminación & 20.37 \\
\hline Falta de vivienda & 29.63 \\
\hline Corrupción & 45.37 \\
\hline Instituciones ineficientes & 29.63 \\
\hline
\end{tabular}

En general, los estudiantes tienen a señalar una mayor cantidad de problemas frente a sus barrios. Las causas de esta mayor "severidad" en juzgar su propio municipio puede tener múltiples causas aún por explorar.

\section{Estudiantes}




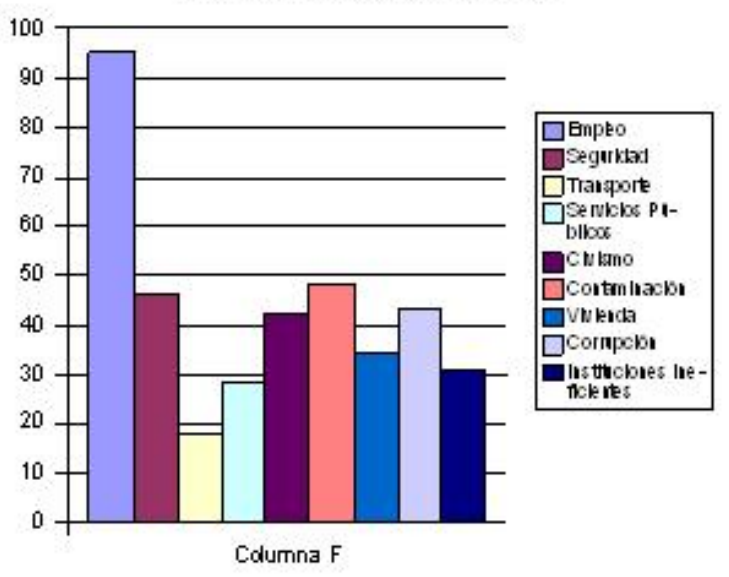

\begin{tabular}{|l|l|}
\hline Problemas sentidos & $\%$ \\
\hline Empleo & 95.2 \\
\hline Inseguridad & 46.4 \\
\hline Transporte & 17.6 \\
\hline Servicios públicos & 28 \\
\hline Falta de civismo & 42.8 \\
\hline Contaminación & 48 \\
\hline Falta de vivienda & 34.4 \\
\hline Corrupción & 43.2 \\
\hline Instituciones ineficientes & 31.2 \\
\hline
\end{tabular}

\section{RESPUESTAS FRENTE A LA INFRACCIÓN DE LA NORMA}

Existe una importante tendencia entre colaboradores de empresas afiliadas de denunciar y dialogar frente a la infracción de la norma con un $67.14 \%$ y $45.71 \%$ que ha acudido a estas vías. Llama la atención la alta tendencia de "dejar las cosas así" entre estudiantes: un $50 \%$ han sido negligentes frente al incumplimiento de la norma de algún conciudadano. Por otro lado, contrasta con que un $36.11 \%$ de los estudiantes declaran haber denunciado situaciones de incumplimiento de la norma.

\section{Empresas}


Respuesta frente a inclumplimiento

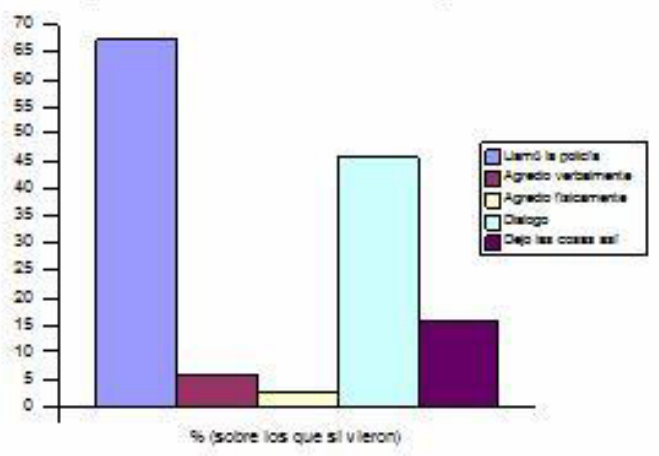

\begin{tabular}{|l|l|}
\hline Vieron infracciones de la norma & $\%$ \\
\hline Sí & 64.81 \\
\hline No & 30.56 \\
\hline $\mathrm{Nr}$ & 4.63 \\
\hline
\end{tabular}

Existe un asunto de atención prioritario entre estudiantes: agresiones verbales. Un $15.28 \%$ han acudido a este medio de corrección frente al incumplimiento de la norma. Dos hipótesis pueden ser planteadas preliminarmente

\section{Estudiantes}

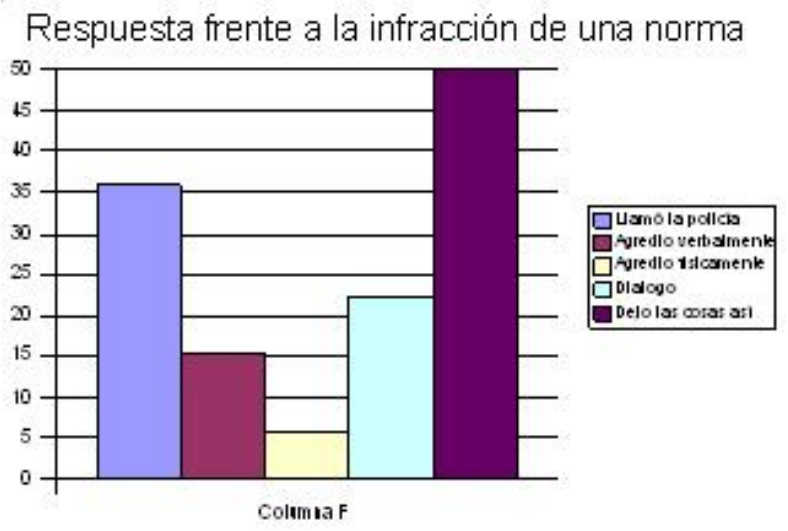

\begin{tabular}{|l|l|}
\hline Vieron infracciones de la norma & $\%$ \\
\hline Sí & 57.6 \\
\hline No & 38.4 \\
\hline $\mathrm{Nr}$ & 4 \\
\hline
\end{tabular}

CONFIANZA Y PRESUNCIÓN DE CONFIANZA CON Y DEL VECINO 
Se presentan importantes contrastes entre la disposición y percepción de la confianza con los vecinos. Los colaboradores de empresas afiliadas afirman en un $78.7 \%$ confiar en sus vecinos. Paralelamente esta visión refleja su posición frente a su presunción de confianza de sus vecinos hacia los encuestados. Así, el $75 \%$ de los colaboradores presumen que sus vecinos confían en ellos.

\section{Empresas}

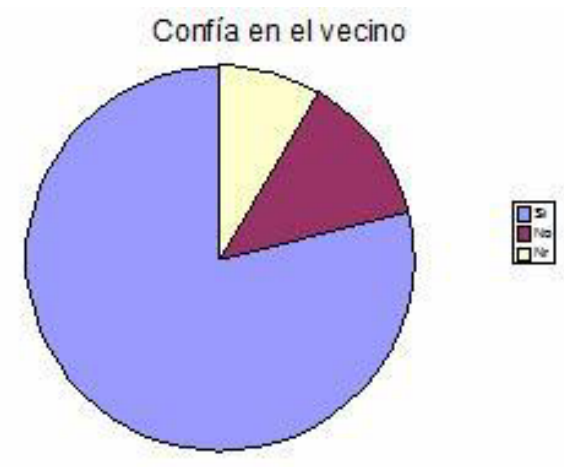

\begin{tabular}{|l|l|}
\hline Confía en el vecino & $\%$ \\
\hline $\mathrm{Si}$ & 78.7 \\
\hline $\mathrm{No}$ & 12.96 \\
\hline $\mathrm{Nr}$ & 8.33 \\
\hline
\end{tabular}

Considera que el vecino confía en él o en ella

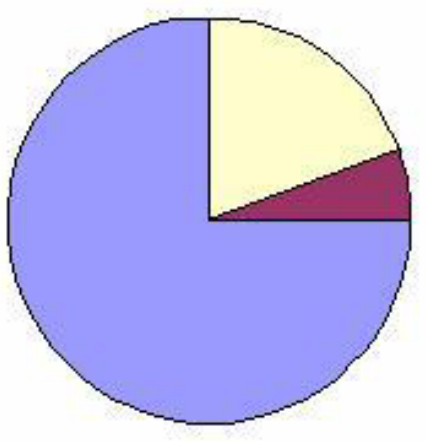

\begin{tabular}{|l|l|}
\hline $\begin{array}{l}\text { Considera que el vecino } \\
\text { confía en él o en ella }\end{array}$ & $\%$ \\
\hline $\mathrm{Si}$ & 75 \\
\hline $\mathrm{No}$ & 5.56 \\
\hline $\mathrm{Nr}$ & 19.44 \\
\hline
\end{tabular}


Por otro lado, el panorama entre los estudiantes es muy diferente. Sólo el $36.8 \%$ de ellos declaran confiar en sus vecinos $\mathrm{y}$, reflejando un comportamiento parecido al de los colaboradores, el $40.8 \%$ consideran que sus vecinos confían en ellos.

\section{Estudiantes}

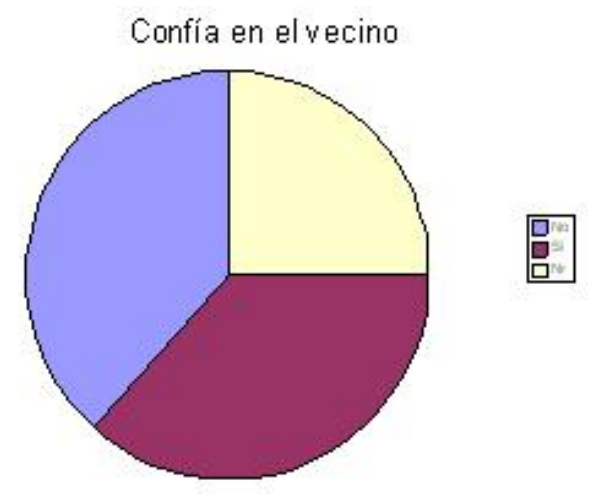

\begin{tabular}{|l|l|}
\hline Confía en el vecino & $\%$ \\
\hline $\mathrm{Si}$ & 36.8 \\
\hline $\mathrm{No}$ & 38.4 \\
\hline $\mathrm{Nr}$ & 24.8 \\
\hline
\end{tabular}

Presume que el vecino confía en el o ella

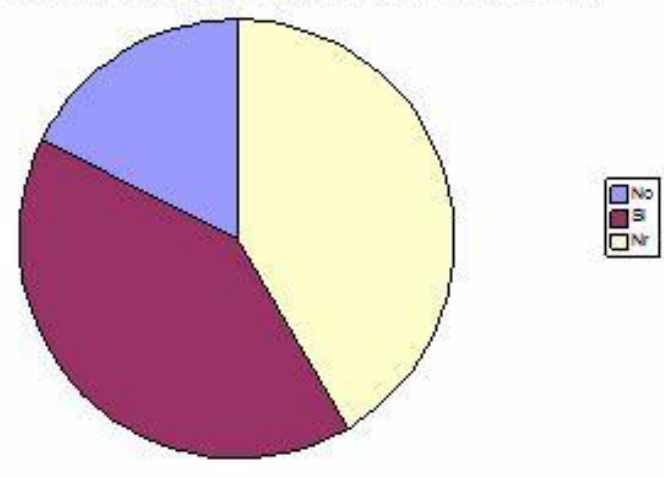

\begin{tabular}{|l|l|}
\hline $\begin{array}{l}\text { Considera que el vecino } \\
\text { confía en él o en ella }\end{array}$ & $\%$ \\
\hline $\mathrm{Si}$ & 40.8 \\
\hline $\mathrm{No}$ & 17.6 \\
\hline $\mathrm{Nr}$ & 41.6 \\
\hline
\end{tabular}

En ambos casos se resalta el hecho de que frente a la presunción de confianza en ambos casos existe una importante franja de opinión que declara " $\mathrm{Nr}$ ", en colaboradores de $19.44 \%$ y entre estudiantes $41.6 \%$. 


\section{PROBLEMAS CON LOS VECINOS}

Entre los colaboradores de empresas afiliadas los dos problemas predominantes son la música y el ruido declarado por el $39.81 \%$ de los encuestados y el chisme con un $35.18 \%$. También, aunque en menor grado, resaltan problemas relacionados con el espacio público o compartido, donde se resaltan problemas con las basuras (19.44\%) y con los animales $(21.26 \%)$.

\section{Empresas}

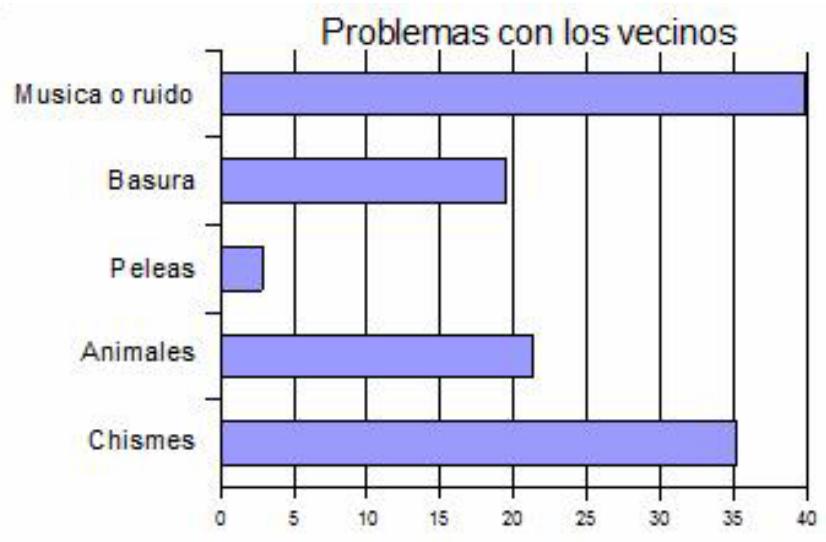

Por el otro lado, los estudiantes resaltan en una proporción parecida al de las empresas afiliadas la música y el ruido con el $36.8 \%$. Los problemas relacionados con espacios públicos o compartidos son reconocidos, pero en menor grado que entre la población adulta, (basura 14.4\%, animales 12.8\%). A diferencia de los colaboradores, las peleas son reconocidas entre esta población como un problema recurrente con los vecinos entre un $11.2 \%$ de los encuestados. El aspecto más llamativo entre los estudiantes es el chisme como el problema más importante con sus vecinos, resaltado por el $77.6 \%$ de los estudiantes.

\section{Estudiantes}

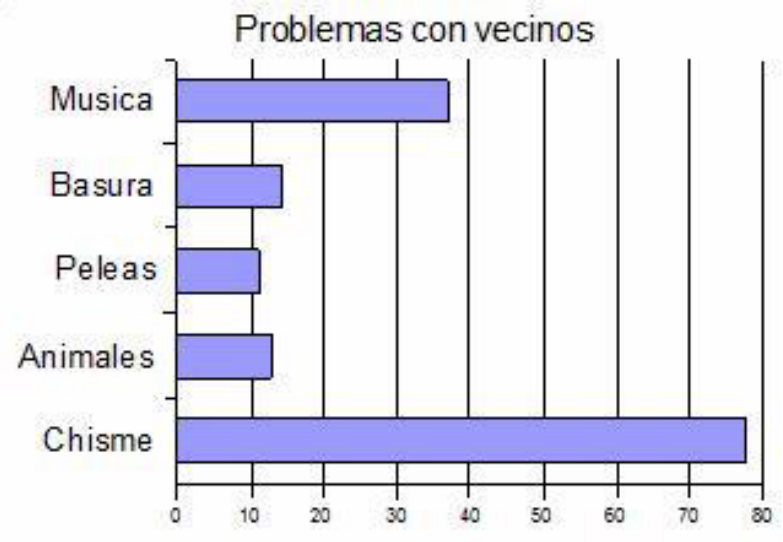

Esta visión de los problemas vecinales entre los estudiantes puede tener relación con la construcción de su identidad en el momento de la vida en el que se encuentran, donde la 
protección del espacio personal y el sentido de intimidad son prioritarios. Ahora bien, la importancia del chisme en ambas poblaciones, sin embargo, puede estar relacionado con una importante proporción de encuestados que viven en municipios pequeños donde es reconocido el impacto del chisme como fuente de conflicto. Lejos de ser una justificación, esto es un llamado de atención sobre la necesidad de trabajo deliberado en torno al chisme en estos municipios.

\section{CONOCIMIENTO DE CÓDIGOS ESCRITOS}

Algunos aspectos frente a esta variable son preocupantes. Después de 15 años de firmada la Constitución Política de Colombia, tenemos entre nuestra población de colaboradores de empresas afiliadas un $10.19 \%$ y, aún más, un $13.6 \%$ de estudiantes que no conocen la Constitución Política. La no lectura de la Constitución es igualmente preocupante: un $42.59 \%$ de colaboradores de empresas afiliadas y un $50.4 \%$ de estudiantes, aun conociéndola, no han leído la Constitución. Es particularmente llamativo que la escuela no esté llevando a mejores niveles de conocimiento a la Constitución.

Igualmente preocupante es el poco conocimiento del reglamento de convivencia ciudadana. Entre colaboradores de empresas un $46.3 \%$ no lo conoce y un $21.3 \%$ aun conociéndolo, no lo han leído. Entre estudiantes, un 53.6\% no lo conoce y un $35.2 \%$ aun conociéndolo, no lo ha leído. El reglamento de convivencia ciudadana tiene mínima apropiación por parte de la ciudadanía. Según indagaciones en grupos focales, se le considera más policial y coercitivo y menos atendiendo a los problemas más cotidianos de convivencia.

\section{Empresas}

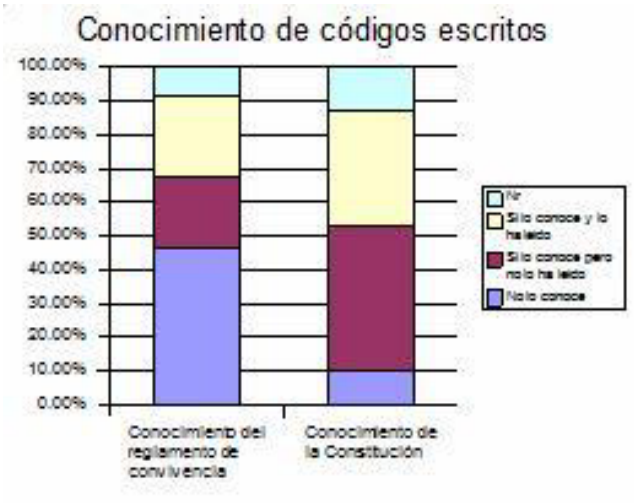

\begin{tabular}{|l|l|l|l|l|}
\hline & $\begin{array}{l}\text { No lo } \\
\text { conoce }\end{array}$ & $\begin{array}{l}\text { Sí lo conoce pero no } \\
\text { lo ha leído }\end{array}$ & $\begin{array}{l}\text { Sí lo ha } \\
\text { leído }\end{array}$ & Nr \\
\hline $\begin{array}{l}\text { Reglamento de } \\
\text { Convivencia }\end{array}$ & 46.3 & 21.3 & 24.07 & 8.33 \\
\hline Constitución Política & 10.19 & 42.59 & 34.26 & 12.96 \\
\hline
\end{tabular}

\section{Estudiantes}




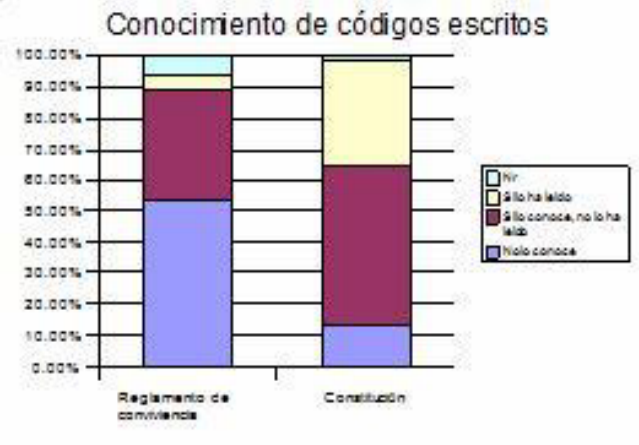

\begin{tabular}{|l|l|l|l|l|}
\hline & $\begin{array}{l}\text { No lo } \\
\text { conoce }\end{array}$ & $\begin{array}{l}\text { Sí lo conoce pero } \\
\text { no lo ha leído }\end{array}$ & $\begin{array}{l}\text { Sí lo ha } \\
\text { leído }\end{array}$ & Nr \\
\hline $\begin{array}{l}\text { Reglamento de } \\
\text { Convivencia }\end{array}$ & 53.6 & 35.2 & 4.8 & 6.4 \\
\hline Constitución Política & 13.6 & 50.4 & 34.4 & 1.6 \\
\hline
\end{tabular}

\section{PERCEPCIONES SOBRE LA NORMA}

En general, la percepción sobre la norma es un acuerdo limitante para ambos casos. Existe muy poca asociación de la norma con la libertad. Para el caso de empresas, esta asociación es del $22.23 \%$ (sumando 1 y 2 del campo de libertad) y para el caso de los estudiantes es del $22.4 \%$.

\section{Empresas}

\begin{tabular}{|l|l|l|l|l|l|l|l|}
\hline$(-)$ & 1 & 2 & 3 & 4 & 5 & $(+)$ & $\mathrm{Nr}$ \\
\hline Gusto & 21.3 & 11.11 & 28.7 & 12.96 & 1.85 & Fastidio & 24.07 \\
\hline Acuerdo & 28.7 & 10.19 & 17.59 & 23.15 & 6.48 & Imposición & 13.89 \\
\hline Libertad & 12.04 & 10.19 & 24.07 & 20.37 & 48 & Limitación & 26.85 \\
\hline Voluntad & 19.44 & 12.04 & 15.74 & 19.44 & 12.96 & Obligación & 20.37 \\
\hline
\end{tabular}

\section{Estudiantes}

\begin{tabular}{|l|l|l|l|l|l|l|l|}
\hline$(-)$ & 1 & 2 & 3 & 4 & 5 & $(+)$ & $\mathrm{Nr}$ \\
\hline Gusto & 16 & 24 & 35.2 & 9.6 & 8 & Fastidio & 7.2 \\
\hline Acuerdo & 36.8 & 9.6 & 22.4 & 16 & 12.8 & Imposición & 3 \\
\hline Libertad & 10.4 & 12 & 33.6 & 20 & 16.8 & Limitación & 7.2 \\
\hline Voluntad & 19.2 & 13.6 & 22.4 & 13.6 & 28 & Obligación & 3.2 \\
\hline
\end{tabular}

\section{JUSTIFICACIÓN DE VIOLACIÓN DE LAS NORMAS}

Si se realiza una media de las respuestas afirmativas para ambas poblaciones, se descubrirá rápidamente una mayor disposición a la violación de la norma entre los estudiantes, con una media del $32.72 \%$ frente al $19.35 \%$ de los colaboradores de 
empresas. La mayor disposición a violar la norma se presenta frente al caso de que la institución o la ley que la aplican son injustas con un $42.52 \%$ entre colaboradores de empresas y un $60.8 \%$ entre estudiantes.

La segunda causa de justificación de la norma entre empresas es el de la necesidad (de abrigo, de alimentos) con un $38.89 \%$. Mientras tanto entre los estudiantes la segunda causa es el desconocimiento de la ley o de la norma con el $51.2 \%$, caso que, sin duda, merece atención en todas las estrategias de competencias ciudadanas.

\section{Empresas}

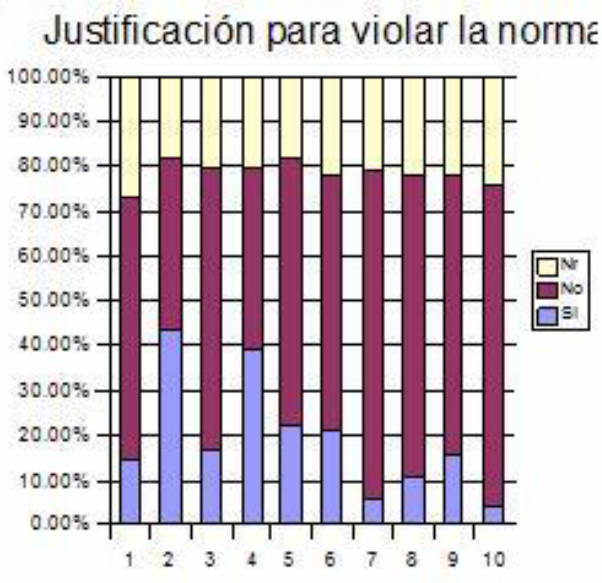

\begin{tabular}{|l|l|l|l|l|}
\hline \multicolumn{1}{|l|}{ Justificación } & $\mathrm{Si}$ & $\mathrm{No}$ & $\mathrm{Nr}$ \\
\hline 1 & No hay violencia & 14.81 & 58.33 & 26.85 \\
\hline 2 & $\begin{array}{l}\text { La institución o la ley que la aplican } \\
\text { son injustas }\end{array}$ & 43.52 & 37.96 & 18.52 \\
\hline 3 & No hay castigo & 16.67 & 62.96 & 20.37 \\
\hline 4 & Hay necesidad & 38.89 & 40.74 & 20.37 \\
\hline 5 & No hace daño a nadie & 22.22 & 59.26 & 18.52 \\
\hline 6 & No se conoce la ley & 21.3 & 56.48 & 22.22 \\
\hline 7 & Es lo acostumbrado & 5.56 & 73.15 & 21.3 \\
\hline 8 & Generaría rechazo social & 11.11 & 66.67 & 22.22 \\
\hline 9 & Para proteger el honor & 15.74 & 62.04 & 22.22 \\
\hline 10 & Es muy provechoso económicamente & 3.7 & 72.22 & 24.07 \\
\hline
\end{tabular}

\section{Estudiantes}




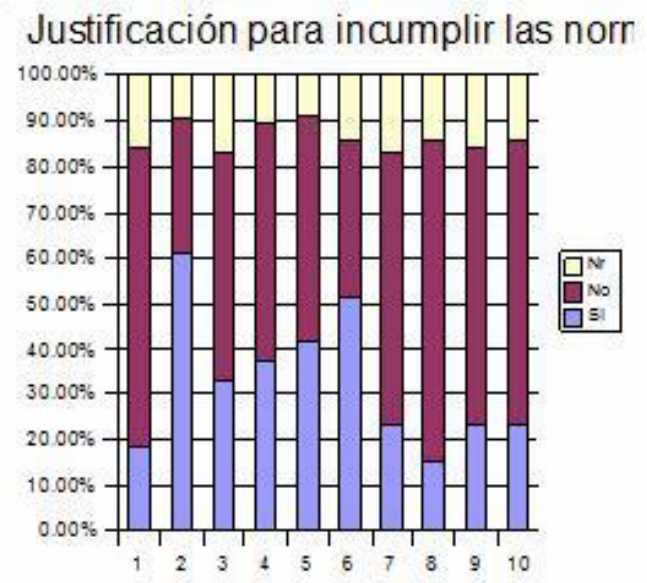

\begin{tabular}{|l|l|l|l|l|}
\hline \multicolumn{1}{|l|}{} & Justificación & $\mathrm{Si}$ & $\mathrm{No}$ & $\mathrm{Nr}$ \\
\hline 1 & No hay violencia & 18.4 & 65.6 & 16 \\
\hline 2 & $\begin{array}{l}\text { La institución o la ley que la aplican son } \\
\text { injustas }\end{array}$ & 60.8 & 29.6 & 9.6 \\
\hline 3 & No hay castigo & 32.8 & 50.4 & 16.8 \\
\hline 4 & Hay necesidad & 37.6 & 52 & 10.4 \\
\hline 5 & No hace daño a nadie & 41.6 & 49.6 & 8.8 \\
\hline 6 & No se conoce la ley & 51.2 & 34.4 & 14.4 \\
\hline 7 & Es lo acostumbrado & 23.2 & 60 & 16.8 \\
\hline 8 & Generaría rechazo social & 15.2 & 70.4 & 14.4 \\
\hline 9 & Para proteger el honor & 23.2 & 60.8 & 16 \\
\hline 10 & Es muy provechoso económicamente & 23.2 & 62.4 & 14.4 \\
\hline
\end{tabular}

Un aspecto importante de atención, particularmente entre los estudiantes, es la disposición alta al incumplimiento de la norma frente a lo acostumbrado (23.2\%), la generación de rechazo social $(15.2 \%)$, para proteger el honor $(23.2 \%)$ y porque es muy provechoso económicamente $(23.2 \%)$.

\section{DISPOSICIÓN A LOS ACUERDOS SEGÚN CONTEXTOS}

El contexto donde menos disposición se encuentra frente al acuerdo -o donde no se tiene reconocido como medida legítima- es el campo de lo ciudadano. El $48.14 \%$ de los encuestados de empresas manifiestan estar "completamente de acuerdo", "de acuerdo" o "indiferentes" frente a que el Alcalde haga acuerdos con el Concejo. Para los estudiantes, la suma de estas posiciones frente a la misma afirmación es de 50.4\%. En el ámbito domestico, por el contrario, existe una disposición mucho mayor hacia los acuerdos.

\section{Empresas}




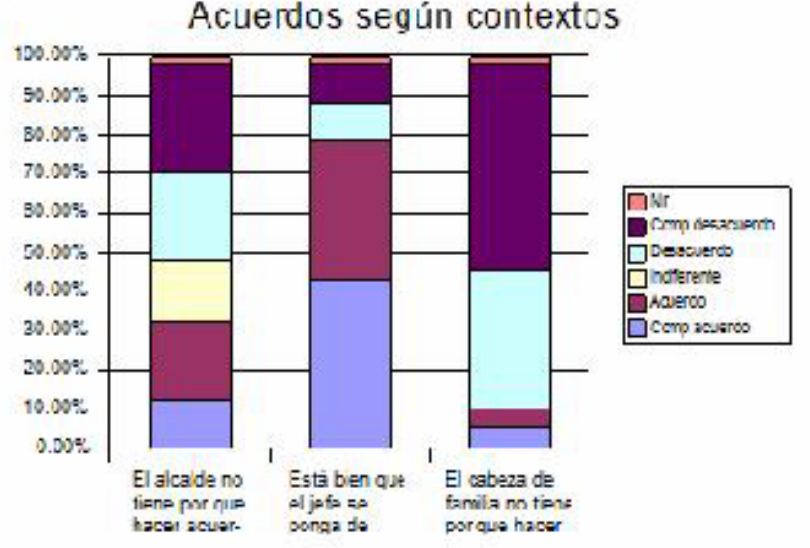

\begin{tabular}{|l|l|l|l|l|l|l|l|}
\hline & $\begin{array}{l}\text { Completamente } \\
\text { de acuerdo }\end{array}$ & $\begin{array}{l}\text { De es } \\
\text { acuerdo }\end{array}$ & $\begin{array}{l}\text { En } \\
\text { indiferente } \\
\text { desacuerdo }\end{array}$ & $\begin{array}{l}\text { Completamente } \\
\text { en desacuerdo }\end{array}$ & $\mathrm{Nr}$ \\
\hline $\begin{array}{l}\text { El Alcalde no } \\
\text { tiene por qué } \\
\text { hacer } \\
\text { acuerdos con } \\
\text { el Concejo }\end{array}$ & 12.96 & 19.44 & 15.74 & 22.22 & 27.78 & 1.85 \\
\hline $\begin{array}{l}\text { Está bien que } \\
\text { el jefe se } \\
\text { ponga de } \\
\text { acuerdo con } \\
\text { sus } \\
\text { colaboradores }\end{array}$ & 43.52 & 35.19 & 0 & 9.26 & 10.19 & 1.85 \\
\hline $\begin{array}{l}\text { El cabeza de } \\
\text { familia } \\
\text { manda y no } \\
\text { tiene por qué } \\
\text { consultar sus } \\
\text { decisiones }\end{array}$ & 5.56 & & & & & & \\
\hline
\end{tabular}

\section{Estudiantes}

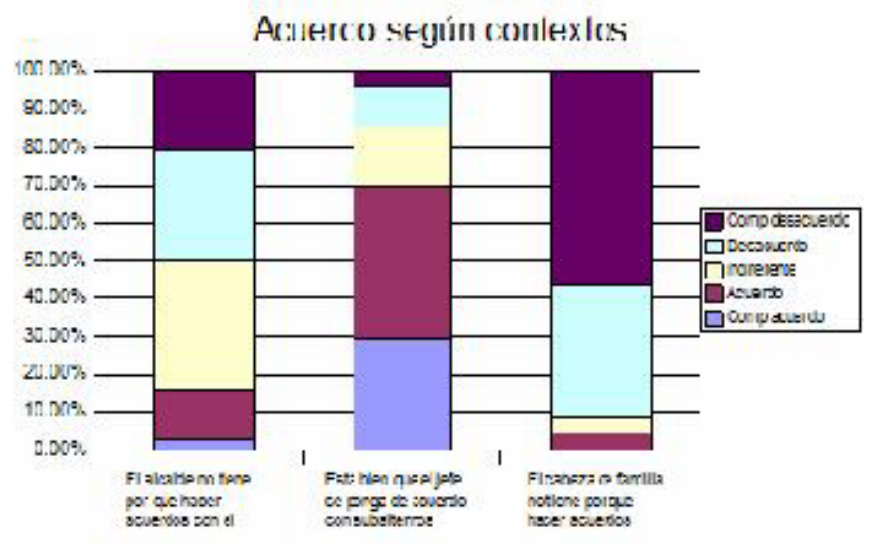




\begin{tabular}{|l|l|l|l|l|l|l|l|}
\hline & $\begin{array}{l}\text { Completamente } \\
\text { de acuerdo }\end{array}$ & $\begin{array}{l}\text { De } \\
\text { acuerdo }\end{array}$ & $\begin{array}{l}\text { Le es } \\
\text { indiferente }\end{array}$ & $\begin{array}{l}\text { En } \\
\text { desacuerdo }\end{array}$ & $\begin{array}{l}\text { Completamente } \\
\text { en desacuerdo }\end{array}$ & $\mathrm{Nr}$ \\
\hline $\begin{array}{l}\text { El Alcalde no } \\
\text { tiene por qué } \\
\text { hacer } \\
\text { acuerdos con } \\
\text { el concejo }\end{array}$ & 3.2 & 12.8 & 34.4 & 28.8 & 20.8 & 0 \\
\hline \begin{tabular}{l|l|l|l|l|} 
Está bien que \\
el jefe se \\
ponga de \\
acuerdo con \\
sus \\
colaboradores
\end{tabular} & 29.6 & & & & & & \\
\hline $\begin{array}{l}\text { El cabeza de } \\
\text { familia } \\
\text { manda y no } \\
\text { tiene por qué } \\
\text { consultar sus } \\
\text { decisiones }\end{array}$ & 0 & 40 & 16 & 10.4 & 4 & \\
\hline
\end{tabular}

\section{DISPOSICIÓN A LOS ACUERDOS}

La visión recurrente frente a la celebración de acuerdos es "confianza con garantías". La situación es más marcada entre los estudiantes donde pedir garantías $(75.2 \%)$ se encuentra por encima de la confianza con los vecinos $(71.2 \%)$. Entre los colaboradores de empresas se mantiene la petición de garantías $(67.59 \%)$, pero en este caso es superado por la confianza de los vecinos (79.63\%)

\section{Empresas}

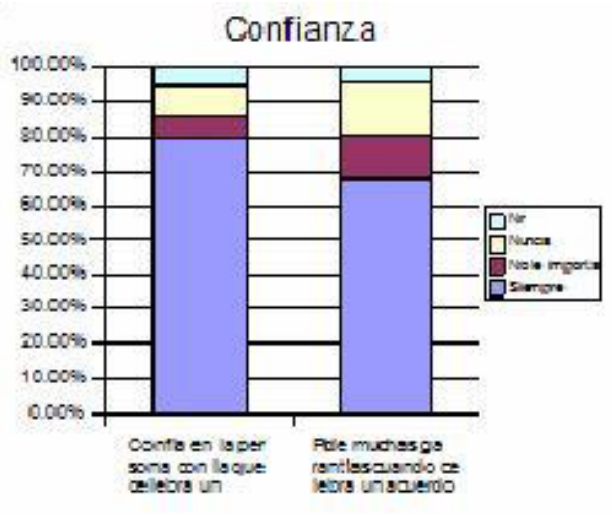

\begin{tabular}{|l|l|l|l|l|}
\hline & Siempre & $\begin{array}{l}\text { No le } \\
\text { importa }\end{array}$ & Nunca & Nr \\
\hline $\begin{array}{l}\text { Confía en la persona con la que } \\
\text { celebra un acuerdo }\end{array}$ & 79.63 & 6.48 & 8.33 & 5.56 \\
\hline
\end{tabular}




\begin{tabular}{|l|l|l|l|l|}
\hline $\begin{array}{l}\text { Pide muchas garantías cuando } \\
\text { celebra acuerdos }\end{array}$ & 67.59 & 12.96 & 14.81 & 4.63 \\
\hline
\end{tabular}

\section{Estudiantes}

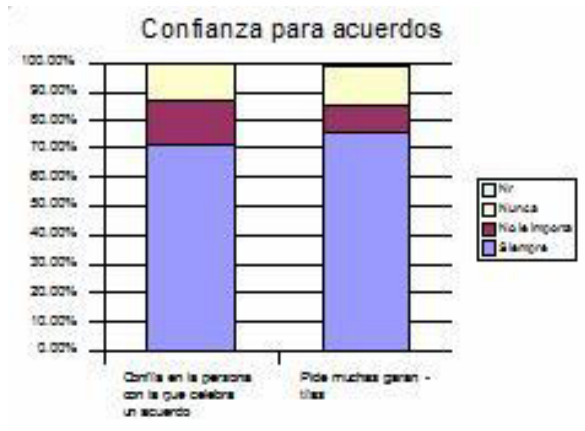

\begin{tabular}{|l|l|l|l|l|}
\hline & Siempre & $\begin{array}{l}\text { No le } \\
\text { importa }\end{array}$ & Nunca & Nr \\
\hline $\begin{array}{l}\text { Confía en la persona con la que } \\
\text { celebra un acuerdo }\end{array}$ & 71.2 & 16 & 12.8 & 0 \\
\hline $\begin{array}{l}\text { Pide muchas garantías cuando } \\
\text { celebra acuerdos }\end{array}$ & 75.2 & 10.4 & 13.6 & 0.8 \\
\hline
\end{tabular}

\section{CONFIANZA EN REPRESENTANTES}

El tema de la confianza en los representantes implica analizar las tres respuestas ("si", "no" y "nr") y no solamente en la respuesta afirmativa. Los mejores niveles de representatividad la tienen los alcaldes y el Presidente. Entre estudiantes la representación es del $44.8 \%$ y $40.8 \%$, respectivamente y entre colaboradores de empresas es del 40.74 y el $50 \%$, respectivamente. Mientras tanto la más baja representatividad la tienen los ediles con un $6.4 \%$ para estudiantes y el $19.44 \%$ entre los grupos de empresas. Sin embargo el caso de las Juntas de Acción Local (JAL) debe ser analizado especialmente dado que en los municipios por fuera de Manizales donde fue aplicada la encuesta no hay comunas y, por ende, no existen JAL. $\stackrel{2}{ }$ Esto explica el gran peso que tiene la respuesta "Nr" $(43.2 \%$ entre estudiantes y $37.96 \%$ entre colaboradores).

Para ambas poblaciones puede ser reconocida una crisis de representación de los cuerpos colegiados. Entre colaboradores de empresas, por ejemplo, todas estas corporaciones tienen una representatividad inferior al $40 \% \mathrm{y}$, de modo aún más grave entre los estudiantes sólo el $17.6 \%$ se sienten representados por los senadores y apenas el 9.6\% con la Cámara de Representantes.

\section{Estudiantes}




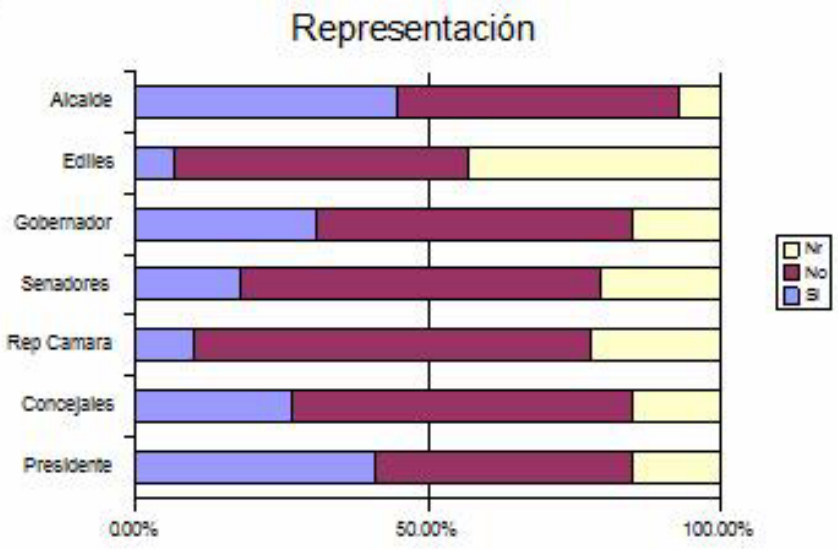

\begin{tabular}{|l|l|l|l|}
\hline & $\mathrm{Si}$ & $\mathrm{No}$ & $\mathrm{Nr}$ \\
\hline Alcalde & 44.8 & 48 & 7.2 \\
\hline Ediles & 6.4 & 50.4 & 43.2 \\
\hline Gobernador & 30.4 & 54.4 & 15.2 \\
\hline Senadores & 17.6 & 61.6 & 20.8 \\
\hline Represent de la Cámara & 9.6 & 68 & 22.4 \\
\hline Concejales & 26.4 & 58.4 & 15.2 \\
\hline Presidente & 40.8 & 44 & 15.2 \\
\hline
\end{tabular}

En ambos casos, el ámbito municipal parece ser una instancia de identificación importante de los representantes. Tanto el Alcalde, como el Concejo tienen representatividades relativamente altas y donde hay más bajas respuestas "Nr", es decir, donde la representación implica alguna posición (negativa o positiva), pero que da poco espacio a la neutralidad.

\section{Empresas}

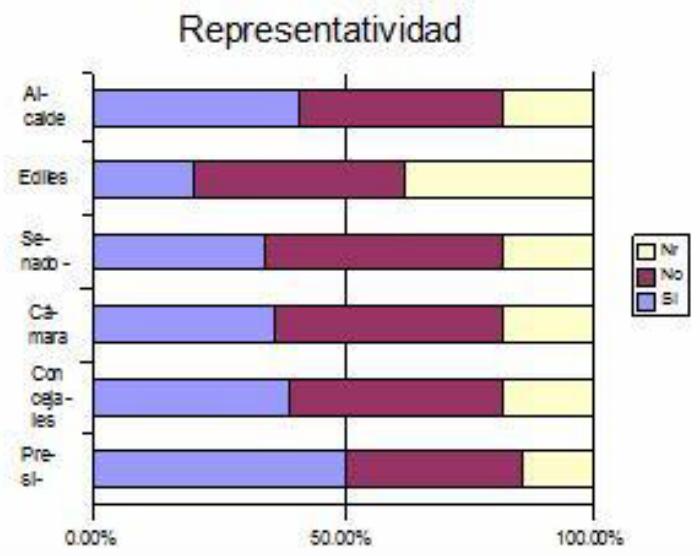

\begin{tabular}{|l|l|l|l|l|}
\hline & $\mathrm{Si}$ & $\mathrm{No}$ & $\mathrm{Nr}$ \\
\hline Alcalde & 40.74 & 40.74 & 18.52 \\
\hline
\end{tabular}




\begin{tabular}{|l|l|l|l|}
\hline Ediles & 19.44 & 42.59 & 37.96 \\
\hline Senadores & 34.26 & 47.22 & 18.52 \\
\hline Represent de la Cámara & 36.11 & 45.37 & 18.52 \\
\hline Concejales & 38.89 & 42.59 & 18.52 \\
\hline Presidente & 50 & 35.19 & 14.81 \\
\hline
\end{tabular}

\section{PARTICIPACIÓN EN ORGANIZACIONES}

La participación es uno de los indicadores que demuestran un panorama más dramático. Sólo el $12.04 \%$ de los colaboradores de empresas y el $7.2 \%$ de los estudiantes pertenecen a una organización comunitaria o política.

\section{Empresas}

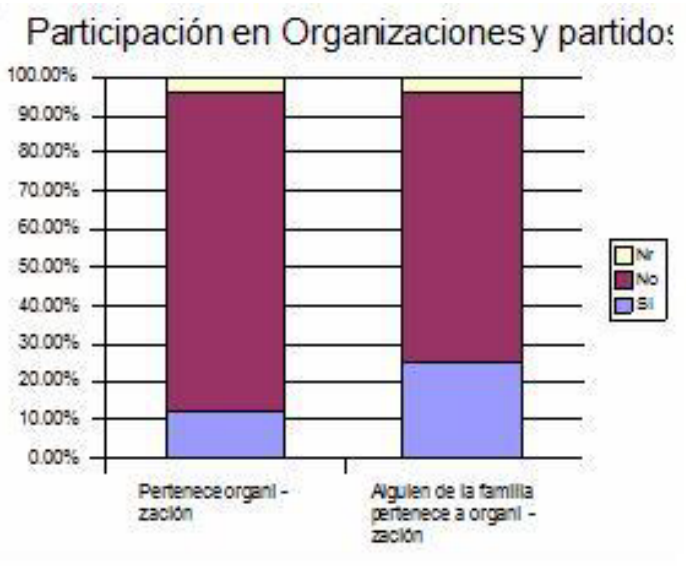

\begin{tabular}{|l|l|l|}
\hline & $\begin{array}{l}\text { Pertenece a una } \\
\text { organización comunal } \\
\text { o política }\end{array}$ & $\begin{array}{l}\text { Alguien de su familia } \\
\text { pertenece a una organización } \\
\text { comunal o política }\end{array}$ \\
\hline $\mathrm{Si}$ & 12.04 & 25 \\
\hline $\mathrm{No}$ & 84.26 & 71.3 \\
\hline $\mathrm{Nr}$ & 3.7 & 3.7 \\
\hline
\end{tabular}

Estudiantes 


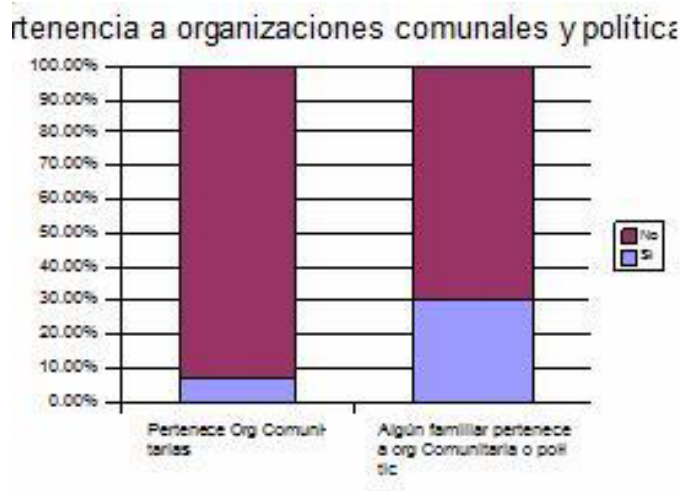

\begin{tabular}{|l|l|l|}
\hline & $\begin{array}{l}\text { Pertenece a una } \\
\text { organización comunal } \\
\text { o política }\end{array}$ & $\begin{array}{l}\text { Alguien de su familia } \\
\text { pertenece a una organización } \\
\text { comunal o política }\end{array}$ \\
\hline $\mathrm{Si}$ & 7.2 & 30.4 \\
\hline $\mathrm{No}$ & 92.8 & 69.6 \\
\hline $\mathrm{Nr}$ & 0 & 0 \\
\hline
\end{tabular}

Este indicador se encuentra por encima del encontrado en el estudio "Pacto por la región" (PNUD, 2004) donde el índice de asociatividad, aunque frente a la participación directa -es decir- la primera de las columnas, coincide.

\section{PERCEPCIÓN FRENTE A ORGANIZACIONES COMUNITARIAS}

Lo anterior contrasta con una relativamente buena percepción frente a las organizaciones comunitarias. Entre los estudiantes, por ejemplo, a pesar de tener más baja asociación con este tipo de organizaciones, se encuentran percepciones más positivas frente a las organizaciones comunitarias con un $40 \%$. Los colaboradores, por el contrario, tienen una buena percepción del $25.22 \%$ frente a estas organizaciones. Parece claro, por lo tanto, que no es la mala percepción de las organizaciones comunitarias lo que explica la poca inclusión en estas. Por el contrario, la poca inclusión de los intereses en las agendas de las organizaciones comunitarias y la permeabilidad de los mismos a conductas clientelistas es un aspecto fundamental señalado por habitantes de los barrios donde hay Juntas de Acción Comunal (JAC) reconocidas y con trayectoria.

\section{Empresas}

\begin{tabular}{|l|l|l|l|l|l|l|l|l|}
\hline$(-)$ & 1 & 2 & 3 & 4 & 5 & $(+)$ & $\mathrm{Nr}$ \\
\hline Ineficientes & 2.78 & 5.56 & 43.52 & 13.89 & 12.96 & Eficientes & 21.3 \\
\hline Autoritarias & 4.63 & 13.89 & 27.78 & 13.89 & 14.81 & Democráticas & 25 \\
\hline Clientelistas & 12.04 & 12.04 & 34.26 & 7.41 & 8.33 & Transparentes & 25.93 \\
\hline Cerradas & 6.48 & 8.33 & 29.63 & 15.74 & 14.81 & Abiertas & 25 \\
\hline
\end{tabular}

\section{Estudiantes}




\begin{tabular}{|l|l|l|l|l|l|l|l|}
\hline$(-)$ & 1 & 2 & 3 & 4 & 5 & $(+)$ & $\mathrm{Nr}$ \\
\hline Ineficientes & 8.8 & 7.2 & 39.2 & 23.2 & 14.4 & Eficientes & 7.2 \\
\hline Autoritarias & 9.6 & 8.8 & 36.8 & 22.4 & 18.4 & Democráticas & 4 \\
\hline Clientelistas & 13.6 & 10.4 & 39.2 & 16.8 & 12 & Transparentes & 8 \\
\hline Cerradas & 4 & 4.8 & 30.4 & 23.2 & 29.6 & Abiertas & 8 \\
\hline
\end{tabular}

\section{TRANSPARENCIA INSTITUCIONAL}

Lo primero observable frente a este aspecto es que no se encuentra una clara división entre entidades públicas y privadas. Los casos más críticos corresponden a las alcaldías y gobernaciones con un 18.52 y 18.52 para empresas y 27.2 y 18.4 para estudiantes.

El primer lugar en términos de confianza en la utilización de los recursos se encuentra en el SENA con percepciones muy positivas entre ambos grupos: $84.26 \%$ para colaboradores de empresas y $82.4 \%$ para estudiantes. Otro aspecto importante de resaltar es la polarización de opiniones en casos como el de la policía, donde prácticamente no existen respuestas "Nr" para ambos grupos, frente al resto de instituciones, donde hay franjas más amplias. Una plausible explicación de esto es el desconocimiento de la labor precisa que desempeña cada una de las instituciones sobre las cuales se indagaron aspectos o, incluso, como en el caso de las JAL's donde no se conoce siquiera su existencia.

\section{Empresas}

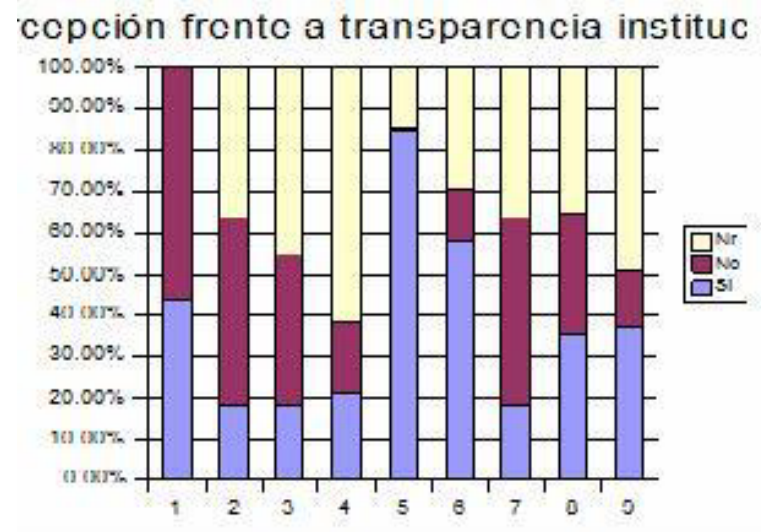

\begin{tabular}{|l|l|l|l|l|}
\hline & & si & No & Nr \\
\hline 1 & Policía & 43.52 & 56.48 & 0 \\
\hline 2 & Alcaldía & 18.52 & 45.37 & 36.11 \\
\hline 3 & Gobernación & 18.52 & 36.11 & 45.37 \\
\hline 4 & JAL & 21.3 & 16.67 & 62.04 \\
\hline 5 & SENA & 84.26 & 0.93 & 14.41 \\
\hline 6 & Cajas de Compensación & 58.33 & 12.04 & 29.63 \\
\hline 7 & Seguro Social & 18.52 & 45.37 & 36.11 \\
\hline
\end{tabular}




\begin{tabular}{|l|l|l|l|l|}
\hline 8 & EPS & 35.19 & 29.63 & 35.19 \\
\hline 9 & Aguas de Manizales & 37.04 & 13.89 & 49.07 \\
\hline
\end{tabular}

\section{Estudiantes}

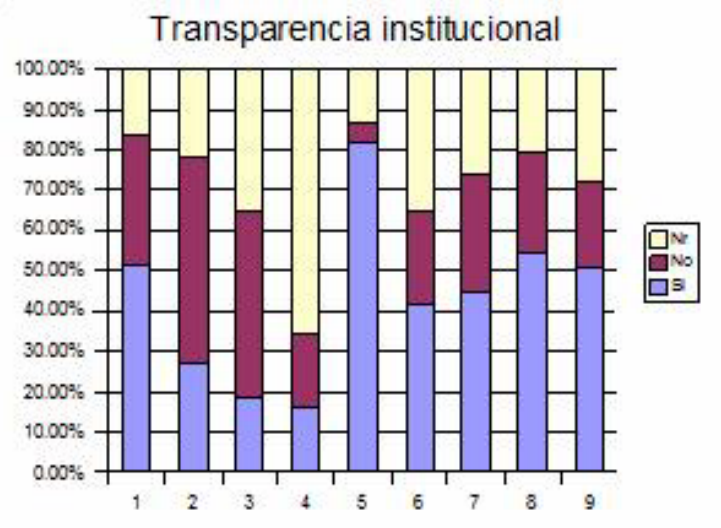

\begin{tabular}{|l|l|l|l|l|}
\hline & & si & No & nr \\
\hline 1 & Policía & 51.2 & 32.8 & 16 \\
\hline 2 & Alcaldía & 27.2 & 50.4 & 22.4 \\
\hline 3 & Gobernación & 18.4 & 4.8 & 65.6 \\
\hline 4 & JAL & 16 & 18.4 & 65.6 \\
\hline 5 & SENA & 82.4 & 4.8 & 12.8 \\
\hline 6 & Cajas de Compensación & 41.6 & 23.2 & 35.2 \\
\hline 7 & Seguro Social & 44.8 & 28.8 & 26.4 \\
\hline 8 & EPS & 54.4 & 24.8 & 20.8 \\
\hline 9 & Aguas de Manizales & 50.4 & 21.6 & 28 \\
\hline
\end{tabular}

\section{PERCEPCIONES FRENTE AL TRIBUTO}

Quizá este sea uno de las hermanas pobres de la ciudadanía. Es, por lo general, un tema no muy grato, pero toda versión de la ciudadanía que la omita, por hacer más bello el discurso, tiene fallas profundas. A la vez percepciones adversas frente al tributo son sintomáticas de cómo está siendo vivida la ciudadanía. En el caso de los colaboradores de empresas afiliadas hay una clara percepción de corrupción en la destinación de los impuestos $(51.85 \%)$ y de resultados no visibles $(62.04 \%)$. En el caso de los estudiantes, los resultados son aún más dicientes: el $60.8 \%$ manifiesta que los impuestos van a manos corruptas y el $68.8 \%$ que los resultados no son visibles

\section{Empresas}


Percepción frente a tributo

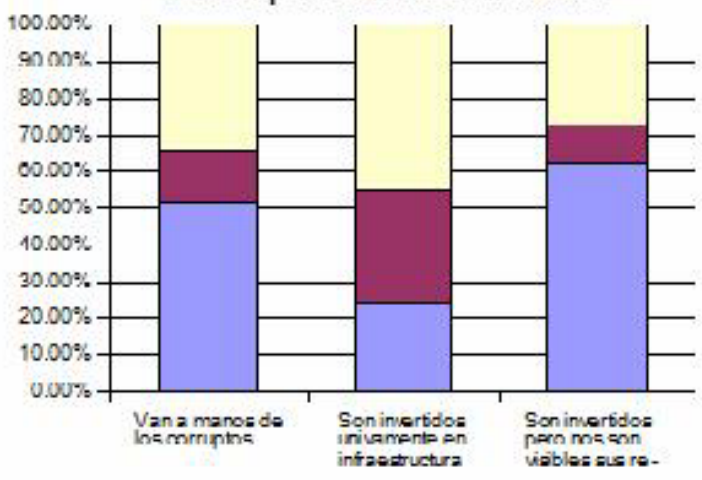

\begin{tabular}{|l|l|l|l|}
\hline & $\mathrm{Si}$ & $\mathrm{No}$ & $\mathrm{Nr}$ \\
\hline $\begin{array}{l}\text { Van a manos de } \\
\text { los corruptos }\end{array}$ & 51.85 & 13.89 & 34.26 \\
\hline $\begin{array}{l}\text { Son invertidos } \\
\text { sólo en } \\
\text { infraestructuras }\end{array}$ & 24.07 & 30.56 & 45.37 \\
\hline $\begin{array}{l}\text { Sus resultados } \\
\text { no son visibles }\end{array}$ & 62.04 & 11.11 & 26.85 \\
\hline
\end{tabular}

\section{Estudiantes}

\section{Tributos}

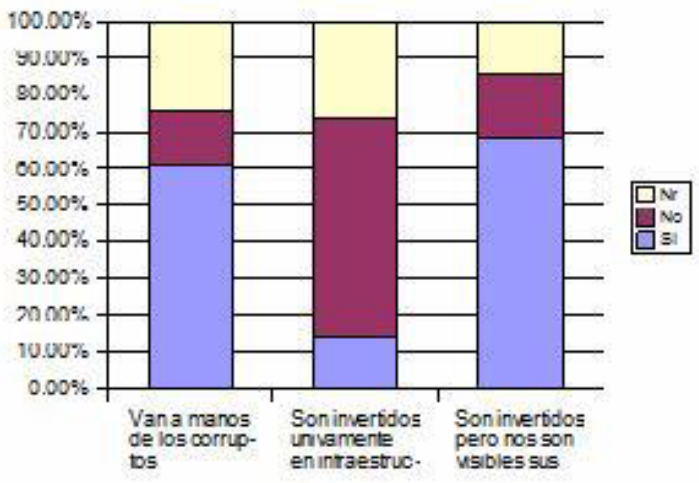

\begin{tabular}{|l|l|l|l|}
\hline & $\mathrm{Si}$ & $\mathrm{No}$ & $\mathrm{Nr}$ \\
\hline $\begin{array}{l}\text { Van a manos de los } \\
\text { corruptos }\end{array}$ & 60.8 & 15.2 & 24 \\
\hline $\begin{array}{l}\text { Son invertidos sólo } \\
\text { en infraestructuras }\end{array}$ & 14.4 & 59.2 & 26.4 \\
\hline $\begin{array}{l}\text { Sus resultados no } \\
\text { son visibles }\end{array}$ & 68.8 & 16.8 & 14.4 \\
\hline
\end{tabular}


Es fundamental poner en marcha ejercicios que lleven a entender cómo se destinan los recursos y cómo funciona el Estado en términos estructurales $\mathrm{y}$, particularmente, financieros.

\section{RESPUESTA FRENTE A LAS AGRESIONES}

Si es necesario resaltar un asunto en un cuadro complicado como este es la disposición a hacer justicia por las propias manos y dejar las cosas así. Las respuestas entre colaboradores se inclinan más a lo legítimo, pero aún queda un nada despreciable $36.11 \%$ que dejarías las cosas así frente a la deuda.

\section{Empresas}

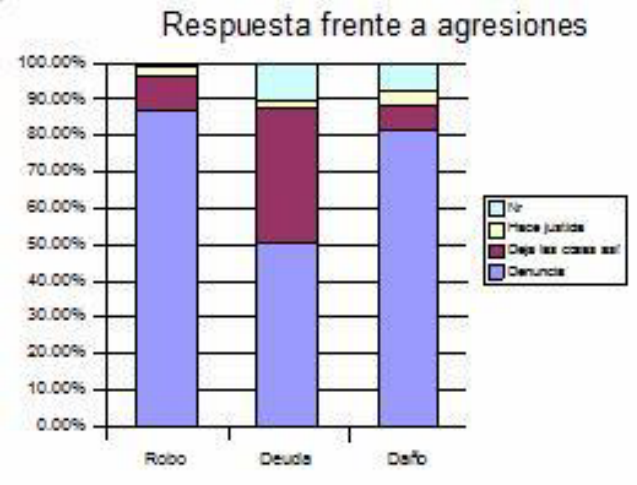

\begin{tabular}{|l|l|l|l|l|}
\hline $\begin{array}{l}\text { Respuesta } \\
\text { frente a }\end{array}$ & Denuncia & $\begin{array}{l}\text { Dejas } \\
\text { las } \\
\text { cosas } \\
\text { así }\end{array}$ & $\begin{array}{l}\text { Hace } \\
\text { justicia }\end{array}$ & $\mathrm{Nr}$ \\
\hline Robo & 86.67 & 9.52 & 2.86 & 0.95 \\
\hline Deuda & 50.93 & 36.11 & 1.85 & 11.11 \\
\hline Daño & 81.49 & 6.48 & 4.63 & 7.41 \\
\hline
\end{tabular}

Frente a los estudiantes el panorama es bien interesante porque refleja una disposición alta hacer justicia por las manos en caso de deuda (20.8\%) y de daño (30.4\%).

\section{Estudiantes}




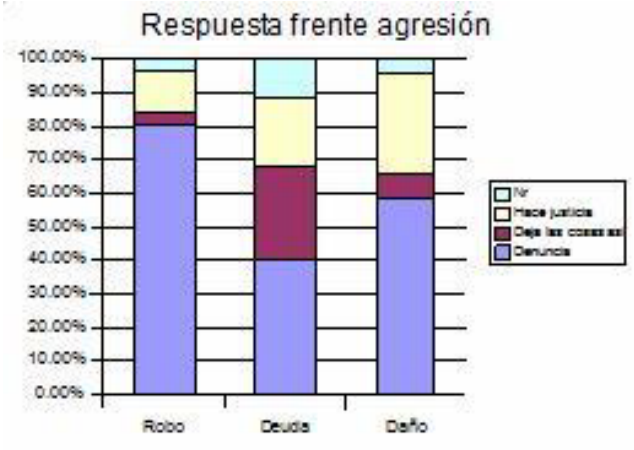

\begin{tabular}{|l|l|l|l|l|}
\hline $\begin{array}{l}\text { Respuesta } \\
\text { frente a }\end{array}$ & Denuncia & $\begin{array}{l}\text { Dejas } \\
\text { las } \\
\text { cosas } \\
\text { así }\end{array}$ & $\begin{array}{l}\text { Hace } \\
\text { justicia }\end{array}$ & $\mathrm{Nr}$ \\
\hline Robo & 80.8 & 3.2 & 12.8 & 3.2 \\
\hline Deuda & 40 & 28 & 20.8 & 11.2 \\
\hline Daño & 58.4 & 7.2 & 30.4 & 4 \\
\hline
\end{tabular}

Es importante resaltar que "dejar las cosas así" y "hacer justicia por la propia cuenta" implican cosas muy distintas de acuerdo a cada uno de los contextos evaluados y pueden incluir el cobrar un dinero o los costos del daño o agredir el agresor en retaliación.

\section{FORMAS ILEGÍTIMAS DE JUSTICIA}

El panorama frente a este aspecto evaluado es preocupante y merece exploraciones cualitativas profundas. Se presenta una significativa tendencia a aceptar formas como la pena de muerte, la autojusticia y la limpieza social como formas de hacer justicia: la media del desacuerdo entre $\mathrm{E}$ es de 52.8 (i.e. El acuerdo es del $47.2 \%$ ) y en CEA es de $48.14 \%$ (i.e. El acuerdo es del 51.86).

Entre CEA existe mucha disposición a aceptar la pena de muerte y la limpieza social y menor disposición a aceptar la justicia por las propias manos. Entre E la aceptación de la pena de muerte es muy baja pero la disposición de la limpieza social es muy alta $(70.4 \%)$

\section{Empresas}

\begin{tabular}{|l|l|l|l|l|l|l|l|}
\hline & $\begin{array}{l}\text { Totalmente } \\
\text { de acuerdo }\end{array}$ & & \multicolumn{3}{|l|}{$\begin{array}{l}\text { Totalmente } \\
\text { en } \\
\text { desacuerdo }\end{array}$} & $\mathrm{Nr}$ \\
\hline & 1 & 2 & 3 & 4 & 5 & \\
\hline $\begin{array}{l}\text { Pena de } \\
\text { muerte }\end{array}$ & 31.48 & 12.04 & 12.04 & 7.41 & 31.48 & 5.56 \\
\hline
\end{tabular}




\begin{tabular}{|l|l|l|l|l|l|l|l|}
\hline Autojusticia & 11.11 & 3.7 & 12.04 & 8.33 & 56.48 & 8.33 \\
\hline $\begin{array}{l}\text { Limpieza } \\
\text { social }\end{array}$ & 25.93 & 4.63 & 20.37 & 10.19 & 30.56 & 8.33 \\
\hline $\begin{array}{l}\text { Tolerancia } \\
\text { extrema }\end{array}$ & 38.89 & 12.04 & 13.89 & 12.96 & 17.59 & 4.63 \\
\hline
\end{tabular}

\section{Estudiantes}

\begin{tabular}{|l|l|l|l|l|l|l|l|}
\hline & $\begin{array}{l}\text { Totalmente } \\
\text { de acuerdo }\end{array}$ & \multicolumn{4}{|c|}{$\begin{array}{l}\text { Totalmente } \\
\text { en } \\
\text { desacuerdo }\end{array}$} & $\mathrm{Nr}$ \\
\hline & 1 & 2 & 3 & 4 & 5 & \\
\hline $\begin{array}{l}\text { Pena de } \\
\text { muerte }\end{array}$ & 12 & 3.2 & 11.2 & 9.6 & 63.2 & 0.8 \\
\hline Autojusticia & 2.4 & 7.2 & 33.6 & 16.8 & 39.2 & 0.8 \\
\hline $\begin{array}{l}\text { Limpieza } \\
\text { social }\end{array}$ & 56 & 4.8 & 8 & 10.4 & 29.2 & 1.6 \\
\hline $\begin{array}{l}\text { Tolerancia } \\
\text { extrema }\end{array}$ & 44 & 9.6 & 20 & 8 & 17.6 & 0.8 \\
\hline
\end{tabular}

\section{TOLERANCIA A LA DIFERENCIA}

Al igual que el anterior, este es un aspecto crítico en los resultados. Se puede encontrar una tendencia a la intolerancia existente en ambos grupos, aunque mucho más pronunciada entre $\mathrm{E}$, con una media de las subpreguntas equivalente al $46.36 \%$, mientras que entre CEA es del $32.11 \%$. Preocupa en ambos casos la tendencia a la intolerancia frente a homosexuales y pacientes con Sida (es decir, frente a conductas sexuales) y, aunque en menor grado, frente a los indígenas.

El espejo de algunas preguntas (es decir, los que aún frente a prácticas condenables manifestaron que no les importaría vivir al lado de quien la hace) es igualmente preocupante, mostrando cierta tendencia a la tolerancia extrema. Por ejemplo, el 31,48\% de CEA que no les importaría vivir junto a un narcotraficante. Es importante trabajar en alguna medida en los procesos formativos frente a lo aceptable e inaceptable en la construcción de normas.

\section{Empresas}




\section{Tipos de personas no aceptadas}

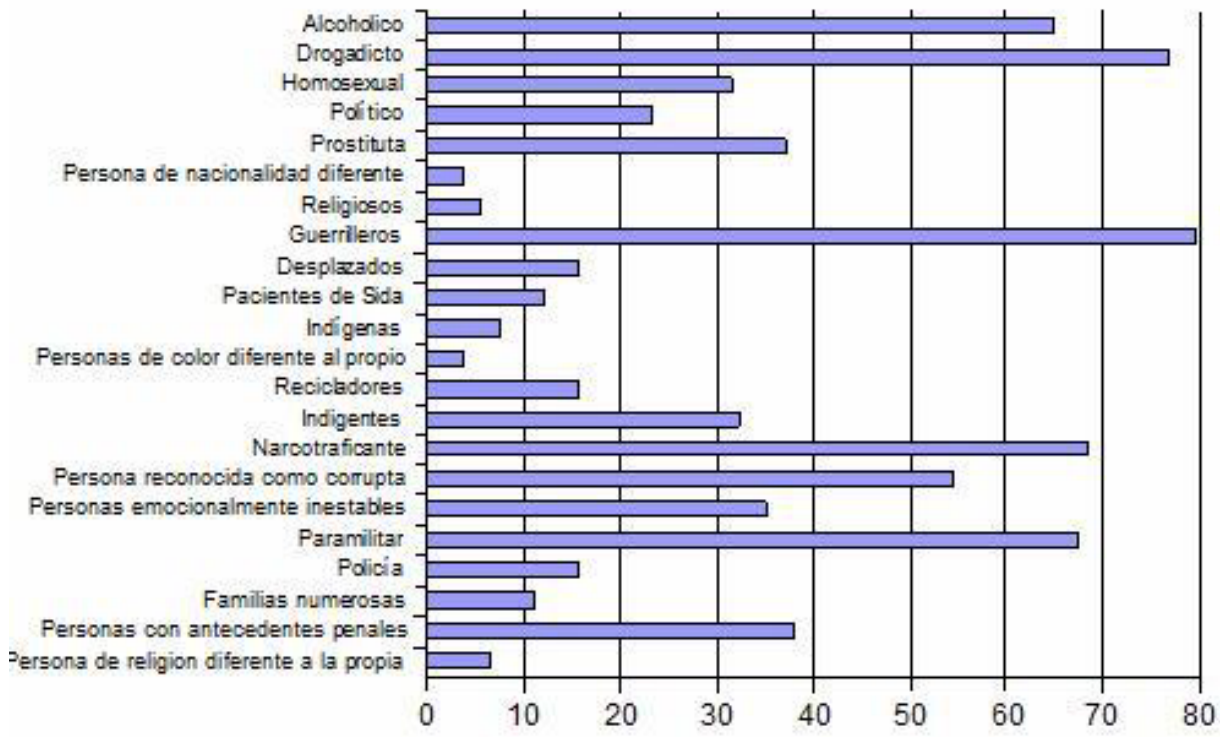

\section{Estudiantes}

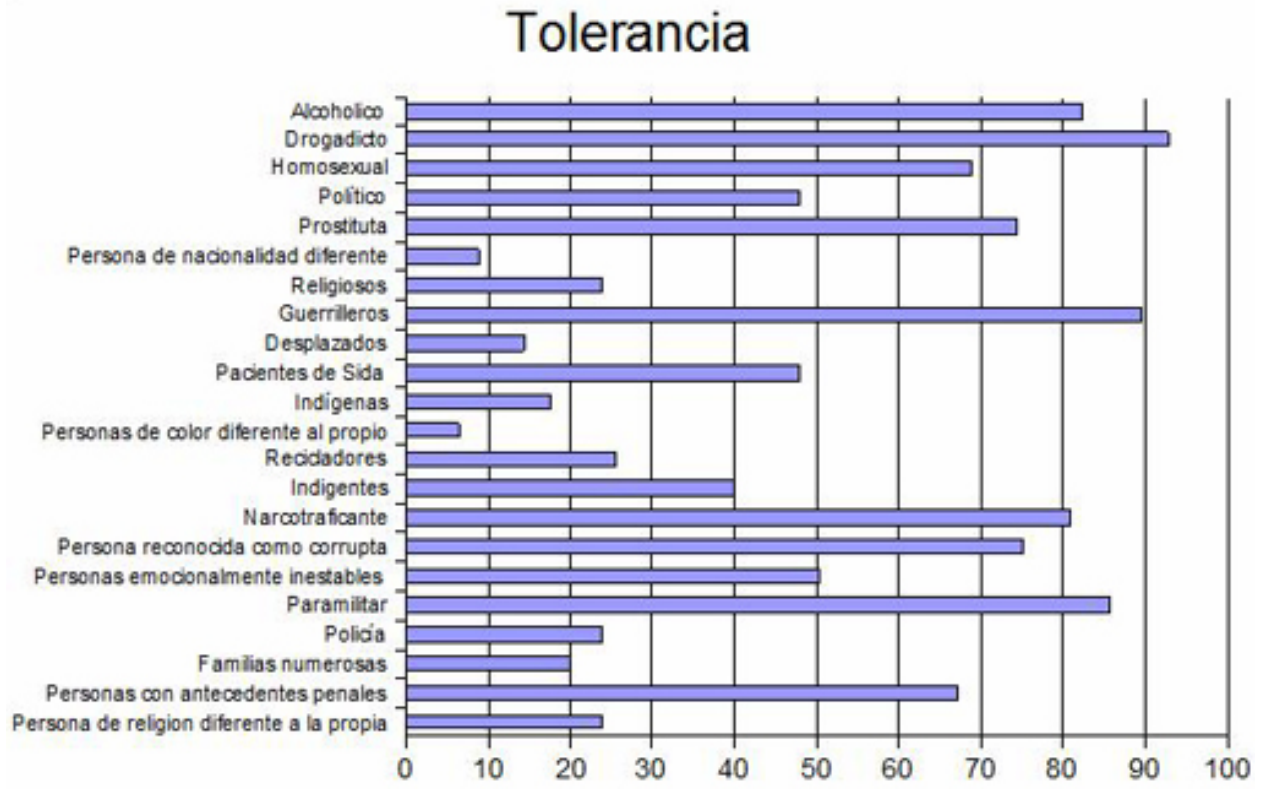

\section{UN CONSOLIDADO FINAL}

A continuación se presenta un consolidado de los indicadores presentados a lo largo de este informe. Los indicadores marcados con rojo son, de acuerdo a los datos, puntos de trabajo prioritario. 


\begin{tabular}{|c|c|c|c|c|c|}
\hline Devoesion & 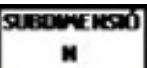 & ASPECTO & EDUCADOR (Ejenplon) & Estudiantes & $\begin{array}{l}\text { Cokaboradore } \\
5 \text { empresas }\end{array}$ \\
\hline \multirow{4}{*}{$\begin{array}{l}\text { NORMAS } \\
\text { LEGALES }\end{array}$} & \multirow{3}{*}{$\begin{array}{l}\text { CULTURA DE } \\
\text { ALLEALIDAD }\end{array}$} & Actitud hacia la ley & $\begin{array}{l}\text { Porcentaje de personas que manifiestan } \\
\text { pdmiración, gusto y/o acuer do frente a } \\
\text { a ley }\end{array}$ & 35 & 31.24 \\
\hline & & $\begin{array}{l}\text { Justificación de la } \\
\text { desobediencia de la } \\
\text { ley }\end{array}$ & $\begin{array}{l}\text { orcentaje de personas que consideran } \\
\text { fue se justifica desobedecer la ley } \\
\text { Euando: es lo acostumbrado, se hace } \\
\text { por luchar contra un régimen injusto, } \\
\text { es muy provechoso económic amente, se } \\
\text { pace por necesidad, es la única } \\
\text { aitternativa para alcanzar sus objetivos, } \\
\text { ptros.) }\end{array}$ & $327:$ & 19.38 \\
\hline & & $\begin{array}{l}\text { Frocedinileatos de } \\
\text { correccibe de } \\
\text { traseresibe deth } \\
\text { porma }\end{array}$ & 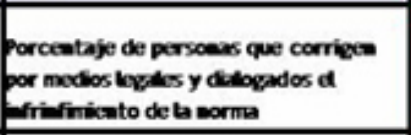 & 29. & 56.42 \\
\hline & $\begin{array}{l}\text { ESPACIO } \\
\text { PÚBLICO }\end{array}$ & $\begin{array}{l}\text { Peroepciones } \\
\text { frente al espacio } \\
\text { pinficos }\end{array}$ & 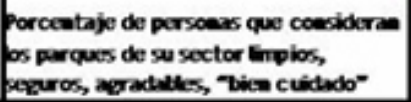 & 72.75 & 56.94 \\
\hline
\end{tabular}

\begin{tabular}{|c|c|c|c|c|c|}
\hline ownesion & 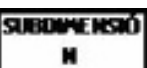 & ASTECIO & EDACADOR (Ejemplos) & Estudiantes & $\begin{array}{l}\text { Colaboradore } \\
\text { s empresas }\end{array}$ \\
\hline & $\begin{array}{l}\text { CONCERTACI } \\
\text { ÓN DE } \\
\text { ACUERDOS }\end{array}$ & $\begin{array}{c}\text { Disposición a } \\
\text { concertar acuerdos }\end{array}$ & $\begin{array}{l}\text { orcentaje de personas dispuestas a } \\
\text { solucionar los probiemas por medio de } \\
\text { acuerdos }\end{array}$ & 70.13 & 72.22 \\
\hline ACUERDOS & $\begin{array}{l}\text { CUMPLIMIENT } \\
\text { O DE } \\
\text { ACUERDOS }\end{array}$ & $\begin{array}{l}\text { Actitud frente al } \\
\text { incumplimiento de } \\
\text { acuerdos y } \\
\text { capacidad para } \\
\text { reparar los } \\
\text { acuerdos } \\
\text { incumplidos }\end{array}$ & $\begin{array}{l}\text { Porcentaje de personas que frente al } \\
\text { ncumptimiento de acuerdos por parte } \\
\text { Je los demás: no hacen nada, piden } \\
\text { gxplicaciones, recurren a la ley, etc. }\end{array}$ & 56.42 & \\
\hline
\end{tabular}

\begin{tabular}{|c|c|c|c|c|c|}
\hline Dowacadin & 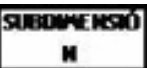 & ASFECID & ETICADOR (Ejemplon) & Estudiantes & $\begin{array}{l}\text { Colaboradore } \\
5 \text { empresas }\end{array}$ \\
\hline & COOPERACIÓ & $\begin{array}{l}\text { Participaciba G } \\
\text { organisacioess } \\
\text { socilles }\end{array}$ & 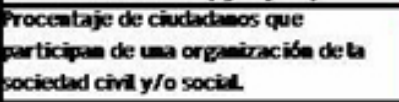 & & $120:$ \\
\hline SOLIDARIDAD & $\begin{array}{l}\text { LOGRO DE } \\
\text { FINES } \\
\text { COMUNES }\end{array}$ & & 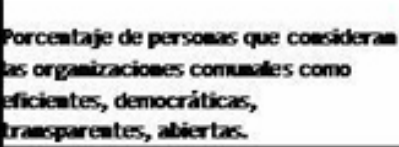 & & 25.22 \\
\hline
\end{tabular}

\begin{tabular}{|c|c|c|c|c|c|}
\hline Duwaesión & 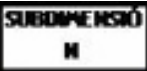 & ASPECIO & EDICADOR (Ejeaplon) & Estudiantes & $\begin{array}{l}\text { Colaboradore } \\
5 \text { empresas }\end{array}$ \\
\hline \multirow{3}{*}{ TOLERANCIA } & \multirow{3}{*}{$\begin{array}{c}\text { ACTITUDES Y } \\
\text { COMPORTAMI } \\
\text { ENTOS } \\
\text { FRENTE A LAS } \\
\text { DIFERENCIAS } \\
\text { RACIALES, } \\
\text { CULTURALES, } \\
\text { RELIGIOSAS Y } \\
\text { POLITICAS }\end{array}$} & $\begin{array}{l}\text { Disposición a } \\
\text { aceptar la } \\
\text { diversidad social, } \\
\text { moral y cultural. } \\
\end{array}$ & $\begin{array}{l}\text { Porcentaje de personas que aceptan } \\
\text { como vecinos a personas con ideas } \\
\text { pouiticas, creencias religiosas, raza, } \\
\text { nctinaciones sexuales, etc., distintas a } \\
\text { as propias }\end{array}$ & 46.36 & 32.11 \\
\hline & & $\begin{array}{l}\text { Respeto por la } \\
\text { familia, los amigos, } \\
\text { los vecinos y } \\
\text { ciudadanos } \\
\text { desconocidos }\end{array}$ & $\begin{array}{l}\text { Porcentaje de personas que agredieron } \\
\text { verbal o fisicamente a ciudadanos por } \\
\text { besacato de las normas }\end{array}$ & 10.42 & $4.2 \mathrm{~g}^{\mathrm{B}}$ \\
\hline & & $\begin{array}{l}\text { Percepción de la } \\
\text { tolerancia } \\
\text { ciudadana }\end{array}$ & $\begin{array}{l}\text { Porcentaje de personas que están en } \\
\text { besacuerdo con la pena de muerte, la } \\
\text { autojusticia, la limpieza social. }\end{array}$ & 52 & 48.14 \\
\hline
\end{tabular}




\begin{tabular}{|c|c|c|c|c|c|}
\hline Dumaesán & 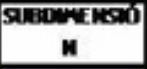 & ASTECIO & IDUCADOR (Ejeaplon) & Estudiantes & $\begin{array}{l}\text { Colaboradore } \\
5 \text { empresas }\end{array}$ \\
\hline \multirow{2}{*}{$\begin{array}{l}\text { IDENTIDAD Y } \\
\text { SENTIDO DE } \\
\text { PERTENENCIA }\end{array}$} & \multirow{2}{*}{$\begin{array}{l}\text { IDENTIDAD } \\
\text { CULTURAL }\end{array}$} & $\begin{array}{c}\text { Sentido de } \\
\text { pertenencia a la } \\
\text { ciudad y al barrio }\end{array}$ & $\begin{array}{l}\text { Porcentaje de personas que consideran } \\
\text { a su ciudad agradable, timpia, segura, } \\
\text { entre otros }\end{array}$ & 52.2 & 53.01 \\
\hline & & & $\begin{array}{l}\text { Porcentaje de personas que consideran } \\
\text { su barrio agradable, timpio, seguro, } \\
\text { entre otros }\end{array}$ & 70.2 & 69.44 \\
\hline
\end{tabular}

\begin{tabular}{|c|c|c|c|c|c|}
\hline Deweesióm & 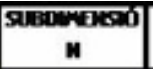 & ASPECIO & EDICADOR (Ejenplos) & Estudiantes & $\begin{array}{l}\text { Colaboradore } \\
\text { s empresas }\end{array}$ \\
\hline \multirow{5}{*}{ CONFIANZA } & \multirow{4}{*}{$\begin{array}{l}\text { CONFIANZA } \\
\text { INTERPERSON } \\
\text { AL EN EL } \\
\text { ÁMBITO } \\
\text { PÚBLICO }\end{array}$} & $\begin{array}{l}\text { Depresantatimictad } \\
\text { de autoridades }\end{array}$ & 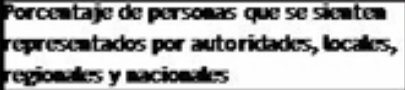 & 25.14 & 36.57 \\
\hline & & $\begin{array}{l}\text { Cosocinilento det } \\
\text { vecino }\end{array}$ & Mimero de vecinos conocidios & & \\
\hline & & $\begin{array}{l}\text { Comfinga co d } \\
\text { recino }\end{array}$ & 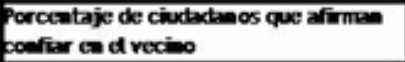 & 38.4 & 78.7 \\
\hline & & $\begin{array}{c}\text { Presuncibe de } \\
\text { confianga ea d } \\
\text { recino }\end{array}$ & $\begin{array}{l}\text { Porcentaje de chuctadlawos que } \\
\text { considera que of vecilo comfia en ellos }\end{array}$ & 40.8 & 75 \\
\hline & $\begin{array}{l}\text { CONFIAALAA } \\
\text { EN LAS } \\
\text { AUTORIDADES } \\
\text { Y LAS } \\
\text { INSTITUCIONE } \\
\text { S PÚBLICAS }\end{array}$ & $\begin{array}{l}\text { Percepcibe sobre } \\
\text { tramsparencia } \\
\text { institucionat }\end{array}$ & 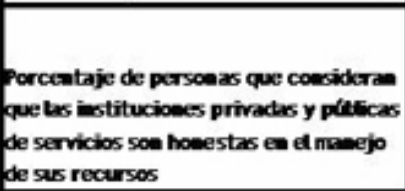 & 42.93 & 37.24 \\
\hline
\end{tabular}

\section{CONCLUSIONES GENERALES}

Además de los énfasis que se infieren a partir de los cuadros anteriores, es importante resaltar los siguientes puntos:

- Es llamativa la persistencia de la respuesta "No Responde" entre el grupo de empresas, particularmente en aspectos relacionados con opiniones personales (Cf. Preguntas de percepción)

- Es necesario continuar el constante sondeo de los indicadores aquí analizados.

- Se hace necesario acompañar el proyecto de análisis cualitativos sobre la estructura y variables involucradas en los problemas que plantea la encuesta.

- Es fundamental cruzar los resultados con otros proyectos institucionales y que se genere apropiación de los indicadores para todo el macro-proceso de Servicios Subsidiados

\section{BIBLIOGRAFÍA}

- LÓPEZ, Natalia. 2004. Procesos de transformación Urbana; Procesos de ocupación y construcción de territorio, Comuna 5 Manizales. Tesis de Grado (Arquitectura), Universidad Nacional de Colombia;: Manizales

- DANE. 1999. Perfil sociodemográfico de Manizales por comunas y barrios. DANE- Gobernacion de Caldas. Secretaría de desarrollo social: Manizales. Nota: los anexos van desde la página 89 a la página 164. 
- MOCKUS, Antanas; BROMBERG, Paul; LONDOÑO, Rocío; PEÑARANDA, Claudia; CASTRO, Carolina; SÁNCHEZ, Efraín. 2004. Guía práctica de cultura ciudadana. Bogotá: Universidad Nacional-PNUD.

- OPS. 1995. Investigación sobre conocimientos, actitudes y prácticas frente a convivencia, dependencia y accidentalidad en escolares de Manizales.

\section{NOTAS}

1. Para todos los casos de indicadores de este tipo, el indicador es la media de las sumas de 4 y 5 en cada una de las filas.

2. De acuerdo a la Reforma Municipal de 1986. 